\title{
Cyclo[6]aramide-Tropylium Charge Transfer Complex as a Colorimetric Chemosensor for Differentiation of Intimate and Loose Ion Pairs
}

Long Chen, Zhiyong Peng, Shuo Liu, Xiaowei Li, Rongzhou Chen, Yi Ren, Wen Feng and Lihua Yuan*

College of Chemistry, Key Laboratory for Radiation Physics and Technology of Ministry of Education, Institute of Nuclear Science and Technology, Key State Laboratory of Biotherapy,

Sichuan University, Chengdu 610064, China

\section{Supporting Information}

\section{Contents}

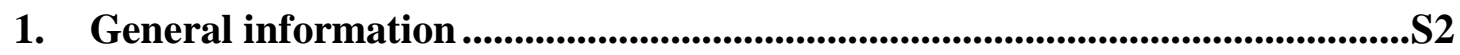

2. Synthesis of compound 3............................................................................................52

3. Characterization of compound 3.............................................................................54

4. Host-guest complexation of 1 and $\mathrm{Tr}^{+}$....................................................................56

4.1 UV-vis spectra ..................................................................................................................56

4.2 2D NOESY spectrum of $1 \supset \mathrm{Tr}^{+}$...........................................................................S6

4.3 MALDI-TOF spectrum of $1 \supset \mathrm{Tr}^{+}$.......................................................................S7

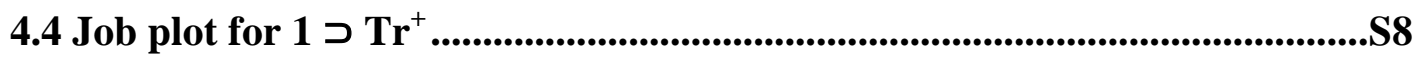

4.5 FT-IR spectra..............................................................................................................S9

4.6 Concentration-dependent ${ }^{1} \mathrm{H}$ NMR spectra of 1 د $\mathrm{Tr}^{+}$...................................S9

4.7 UV-vis titration of $1 \supset \mathrm{Tr}^{+}$.......................................................................................S10

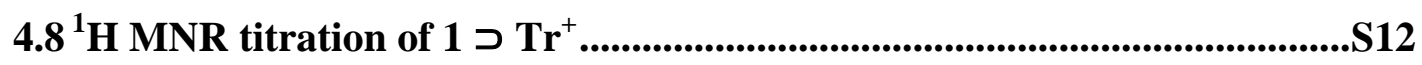

5. Host-guest complexation of 3 and $\mathrm{Tr}^{+}$................................................................S14

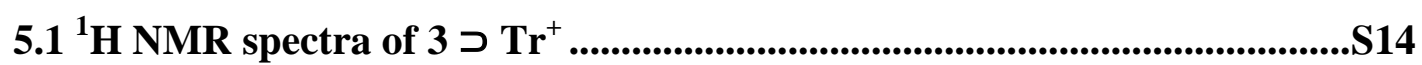

$5.2 \mathrm{UV}$-vis titration of $3 \mathrm{~S} \mathrm{Tr}^{+}$.................................................................................S15

6. UV-vis spectra of $1 \supset \operatorname{Tr}^{+}$after addition of DBuA...........................................S17

7. Host-guest complexation of 1 and DBuA...........................................................S19

7.1 Host-guest complexation of 1 and $4 a$....................................................................S19

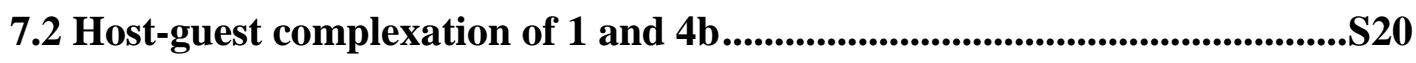




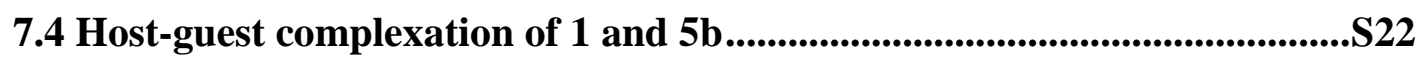

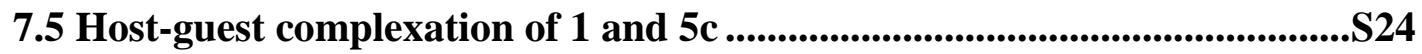

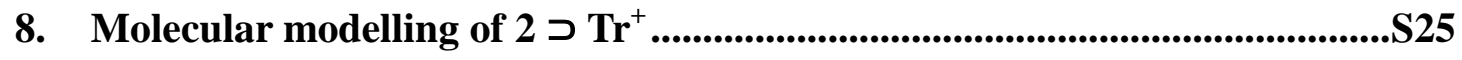

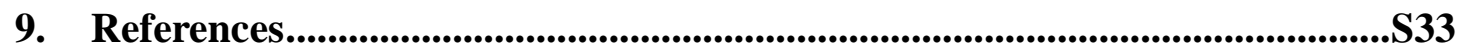

\section{General information}

All chemicals were obtained from commercial suppliers and were used as received without further purification unless otherwise noted. $\mathrm{CH}_{2} \mathrm{Cl}_{2}$ was dried over $\mathrm{CaH}_{2}$. Solvents for extraction were reagent grade. $\mathrm{CDCl}_{3}$ was from Cambridge Isotope Laboratories (CIL) and acetonitrile- $d_{3}$ was supplied by Aldrich.

${ }^{1} \mathrm{H}$ NMR and ${ }^{13} \mathrm{C}$ NMR spectra were recorded on Bruker AVANCE AV II-400 MHz $\left({ }^{1} \mathrm{H}: 400 \mathrm{MHz} ;{ }^{13} \mathrm{C}: 101 \mathrm{MHz}\right)$. 2D NOESY spectra were recorded on Bruker AVANCE AV II-600 MHz. Chemical shifts are reported in $\delta$ values in ppm using tetramethylsilane (TMS) as internal standard and coupling constants $(J)$ are denoted in Hz. Multiplicities are denoted as follows: $\mathrm{s}=$ singlet, $\mathrm{d}=$ doublet, $\mathrm{t}=$ triplet, and $\mathrm{m}=$ multiplet. High resolution mass spectrometer (HRMS) data were obtained by WATERS Q-TOF Premier. MALDI-TOF MS spectra were recorded on Bruker Autoflex III MS spectrometer. UV-vis spectra were measured by SHIMADZU UV-2450. Fourier transform Infrared (FT-IR) data were collected by a Thermo Nicolet NEXUS 670 FT-IR spectrophotometer.

\section{Synthesis of compound 3}

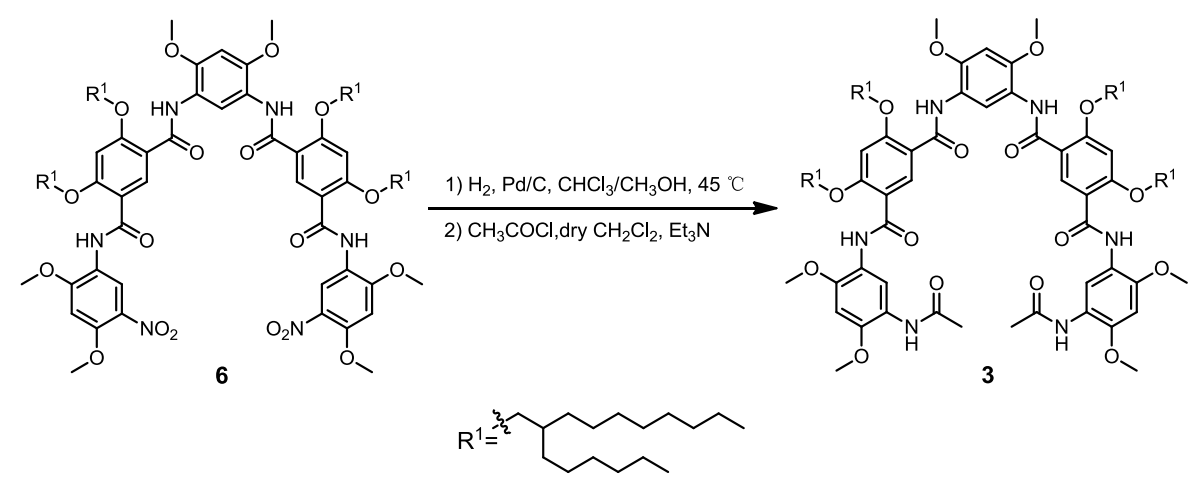

Scheme S1. Synthetic route of 3 . 
A mixture of compound 6 (200 mg, $0.112 \mathrm{mmol})$ and $10 \% \mathrm{Pd} / \mathrm{C}(50 \mathrm{mg})$ was added to $\mathrm{CHCl}_{3} / \mathrm{CH}_{3} \mathrm{OH}(5 / 1, \mathrm{v} / \mathrm{v}, 30 \mathrm{~mL})$. The solution was purged with hydrogen three times and stirred in hydrogen atmosphere for $24 \mathrm{~h}$ at $45{ }^{\circ} \mathrm{C}$. The suspension liquid was filtered to remove $\mathrm{Pd} / \mathrm{C}$ and the gray filtrate was evaporated. The obtained gray powder was dissolved in dry $\mathrm{CH}_{2} \mathrm{Cl}_{2}$ and evaporated again to remove any residual $\mathrm{CH}_{3} \mathrm{OH}$. After dried under vacuum at $45{ }^{\circ} \mathrm{C}$ for half an hour, the pentamer amine was dissolved in dry $\mathrm{CH}_{2} \mathrm{Cl}_{2}$ and stirred with triethylamine (113 $\mathrm{mg}, 1.12 \mathrm{mmol}$ ) for $5 \mathrm{~min}$. Acetyl chloride (43.9 mg, $0.560 \mathrm{mmol}$ ) was added dropwise and the solution was stirred for $3 \mathrm{~h}$ at room temperature. The beige reaction solution was washed with diluted hydrochloric acid $(1 \mathrm{M}, 2 \times 20 \mathrm{~mL})$ and saturated brine $(3 \times 20 \mathrm{~mL})$, respectively. The organic layer was dried over anhydrous $\mathrm{Na}_{2} \mathrm{SO}_{4}$ and the solvent was evaporated to afford a beige solid. The crude product was dissolved in a minimum amount of $\mathrm{CH}_{2} \mathrm{Cl}_{2}$ and then ethyl acetate was added dropwise until no more solid generated. The suspension liquid was filtered and the obtained solid was recrystallized with $\mathrm{CH}_{2} \mathrm{Cl}_{2} / \mathrm{CH}_{3} \mathrm{OH}$ twice to provide 3 as a white solid (154 mg, 75.9\%). ${ }^{1} \mathrm{H}$ NMR (400 $\mathrm{MHz}, \mathrm{CDCl}_{3} / \mathrm{CD}_{3} \mathrm{CN}, 1 / 1$, v/v, $298 \mathrm{~K}$ ) $\delta 9.77$ (s, 2H), 9.74 (s, 2H), 9.29 (s, 1H), 8.99 (d, 4H), 7.77 (s, 2H), 6.70 (s, 2H), 6.68 (s, 1H), 6.65 (s, 2H), 4.21-4.19 (m, 8H), 3.93 (s, 6H), 3.90 (s, 6H), 3.89 (s, 6H), 2.08-2.01 (m, 4H), 1.51-1.23 (m, 96H), 0.88-0.82 $(\mathrm{m}, 24 \mathrm{H}) ;{ }^{13} \mathrm{C}$ NMR $\left(101 \mathrm{MHz}, \mathrm{CDCl}_{3}, 298 \mathrm{~K}\right) \delta 168.07,162.25,162.20,160.18$, $160.11,147.25,146.93,146.78,137.68,120.55,120.42$, 119.63, 118.69, 117.50, 115.82, 96.61, 95.24, 72.79, 56.07, 55.94, 37.69, 31.89, 31.86, 31.09, 30.02, 29.67, 29.63, 29.34, 26.75, 26.70, 24.27, 22.67, 14.11, 14.10, 14.08; ESI-HRMS (m/z) calcd. for $\mathrm{C}_{108} \mathrm{H}_{172} \mathrm{~N}_{6} \mathrm{O}_{16}[\mathrm{M}+\mathrm{Na}]^{+}$1833.2761, found 1833.3230.

Cyclo[6]aramide 1 was synthesized following a literature procedure. ${ }^{\mathrm{S}}$

Tropylium tetrafluoroborate, $\mathbf{4 a}$ and $\mathbf{5 a}$ were obtained from commercial sources. $\mathbf{4} \mathbf{b}^{\mathrm{S} 2}, \mathbf{5} \mathbf{b}^{\mathrm{S} 3}$ and $\mathbf{5} \mathbf{c}^{\mathrm{S} 3}$ were synthesized according to literature procedures. 


\section{Characterization of compound 3}

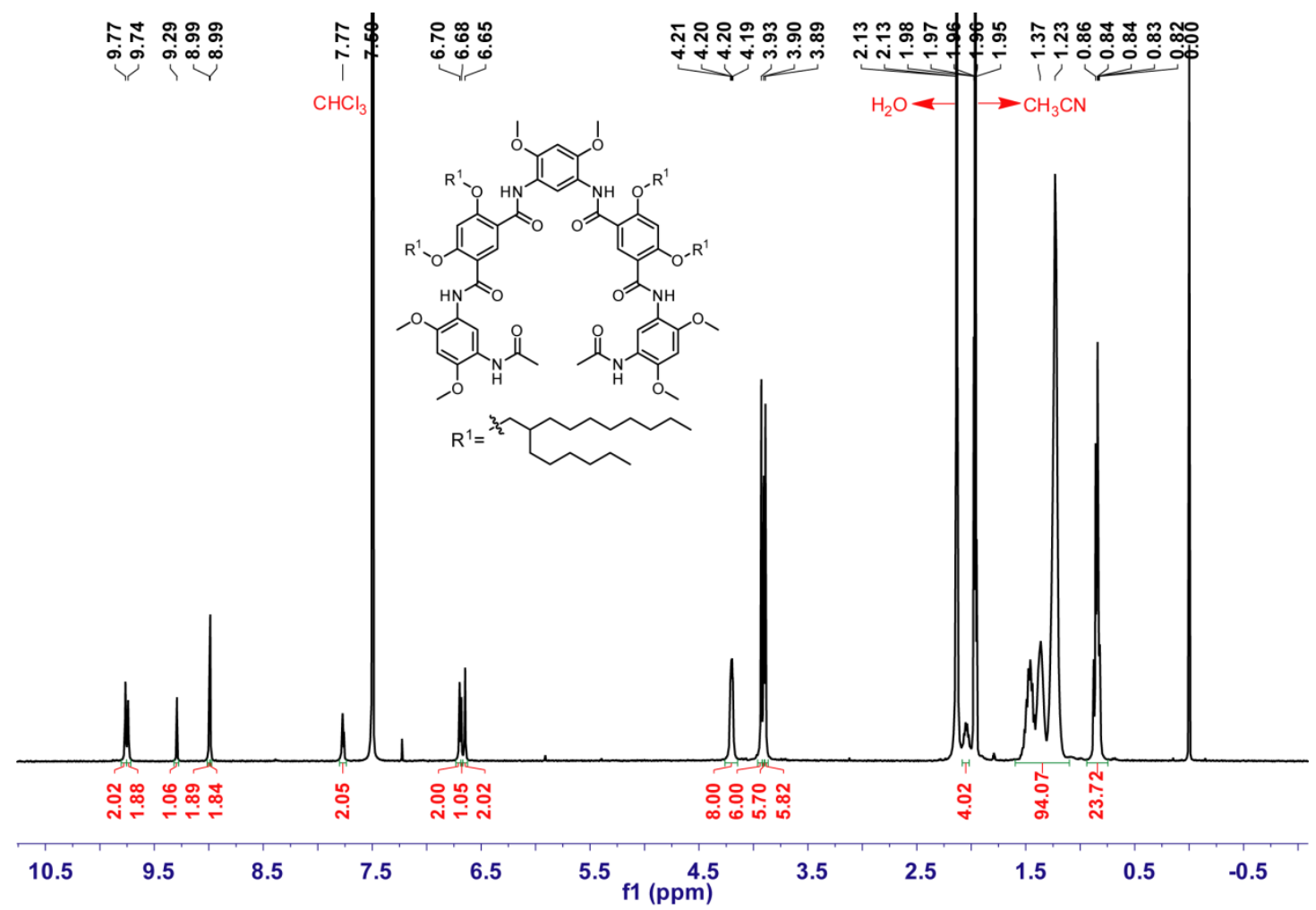

Figure S1. ${ }^{1} \mathrm{H}$ NMR spectrum ( $\left.400 \mathrm{MHz}, \mathrm{CDCl}_{3} / \mathrm{CD}_{3} \mathrm{CN}, 1 / 1, \mathrm{v} / \mathrm{v}\right)$ of $\mathbf{3}$ at $298 \mathrm{~K}$.

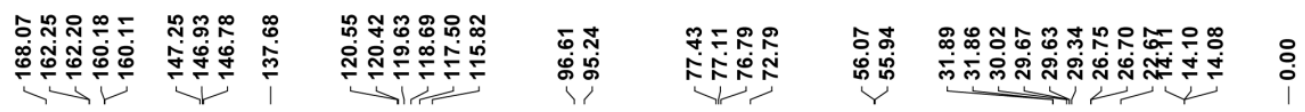
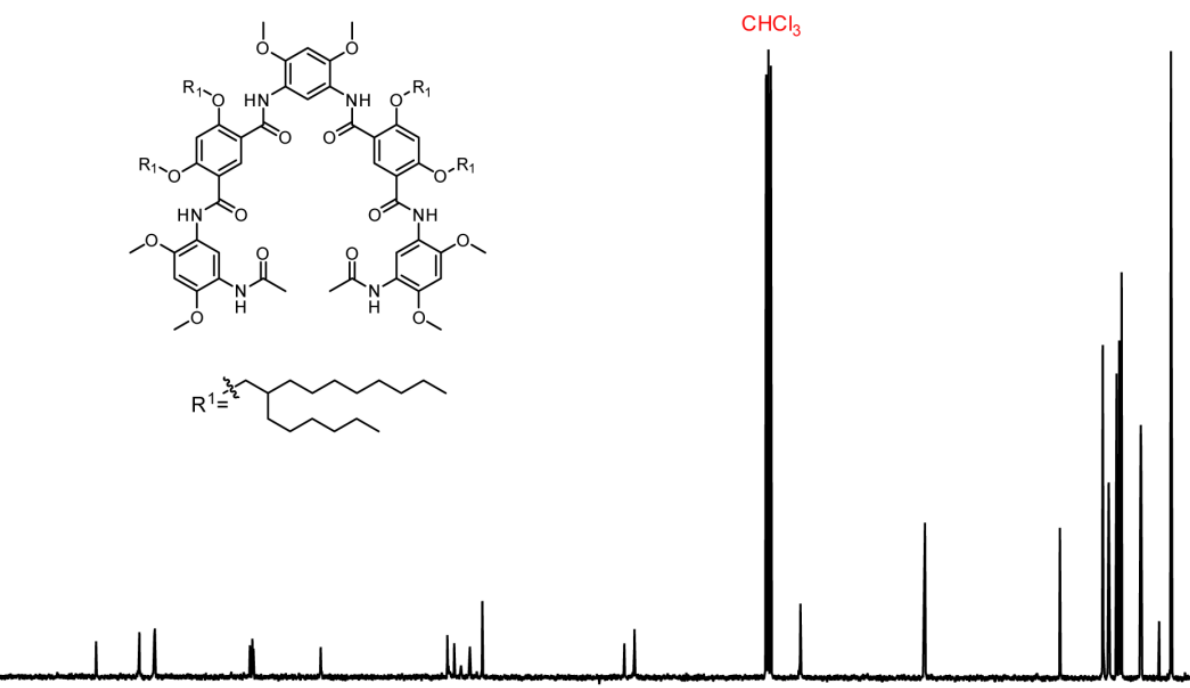

$\begin{array}{llllllllllllllllllll}180 & 170 & 160 & 150 & 140 & 130 & 120 & 110 & 100 & 90 & 80 & 70 & 60 & 50 & 40 & 30 & 20 & 10 & 0 & -10\end{array}$

Figure S2. ${ }^{13} \mathrm{C}$ NMR spectrum $\left(101 \mathrm{MHz}, \mathrm{CDCl}_{3}\right)$ of $\mathbf{3}$ at $298 \mathrm{~K}$. 


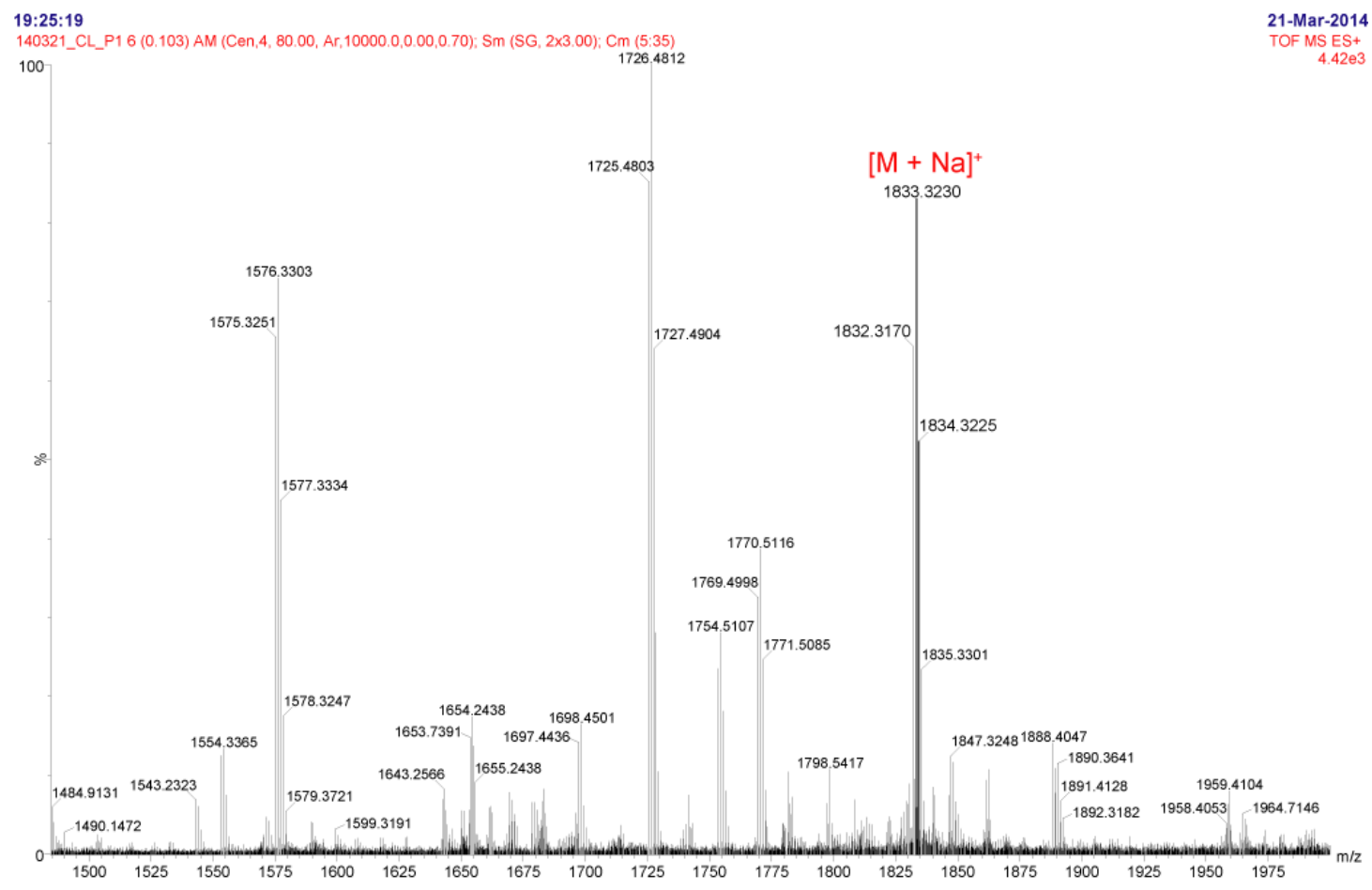

Figure S3. ESI-HRMS spectrum of 3. 


\section{Host-guest complexation of 1 and $\mathrm{Tr}^{+}$}

\subsection{UV-vis spectra}

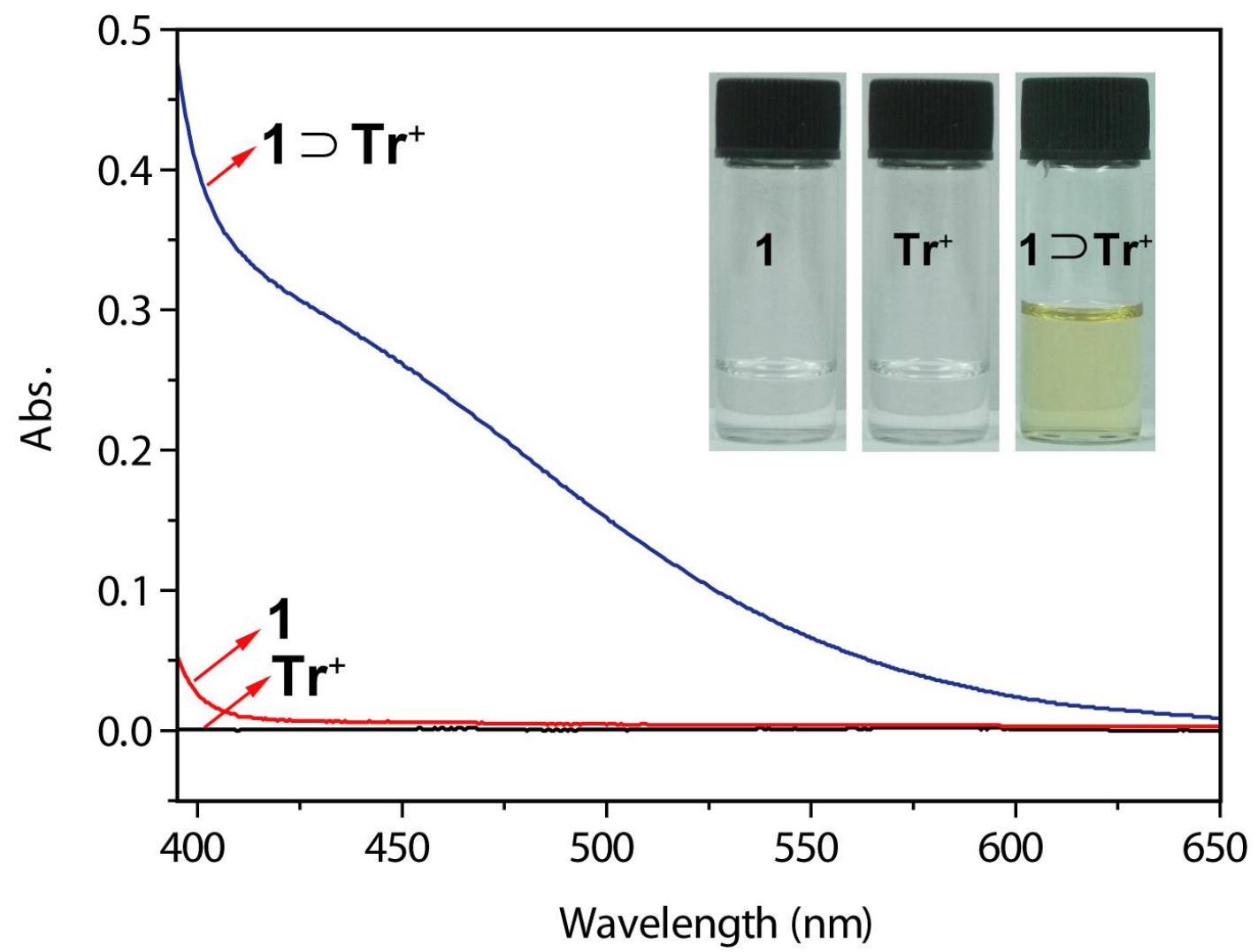

Figure S4. UV-vis spectra of $\mathbf{1}, \mathbf{T r}^{+}$and $\mathbf{1} \supset \mathbf{T r}^{+}$(1 mM for each) in $\mathrm{CHCl}_{3} / \mathrm{CH}_{3} \mathrm{CN}$ $(1 / 1, \mathrm{v} / \mathrm{v})$. Inserted images show the color change.

\subsection{D NOESY spectrum of $1 \supset \operatorname{Tr}^{+}$}

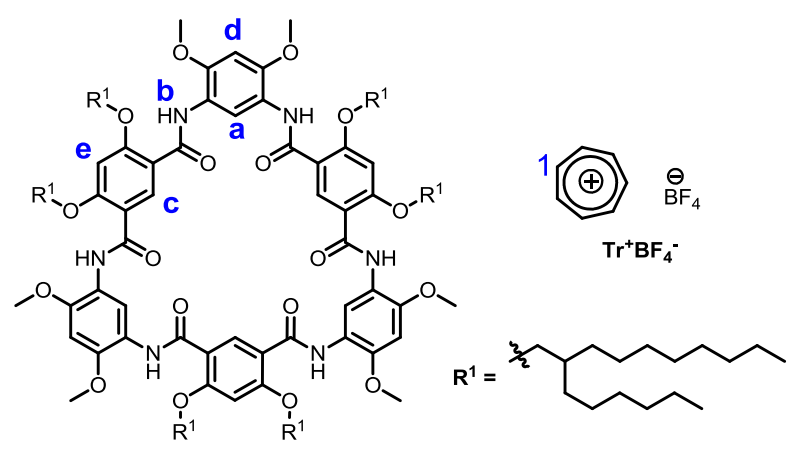




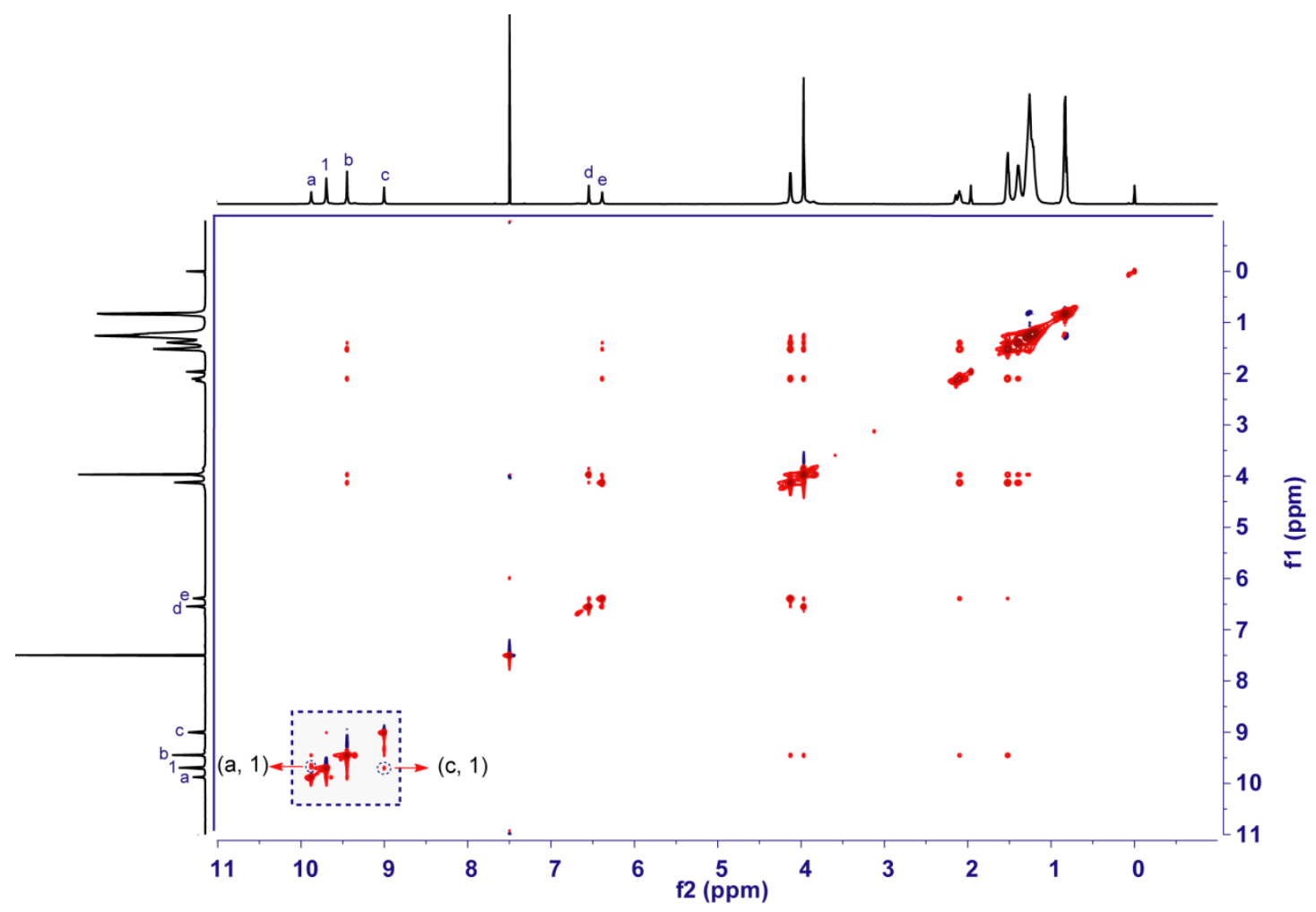

Figure S5. 2D NOESY spectrum $\left(600 \mathrm{MHz}, \mathrm{CDCl}_{3} / \mathrm{CD}_{3} \mathrm{CN}, 1 / 1\right.$, v/v) between 1 and $\mathbf{T r}^{+}$(20 $\mathrm{mM}$ for each) at $298 \mathrm{~K}$.

\subsection{MALDI-TOF spectrum of $1 \supset \mathrm{Tr}^{+}$}

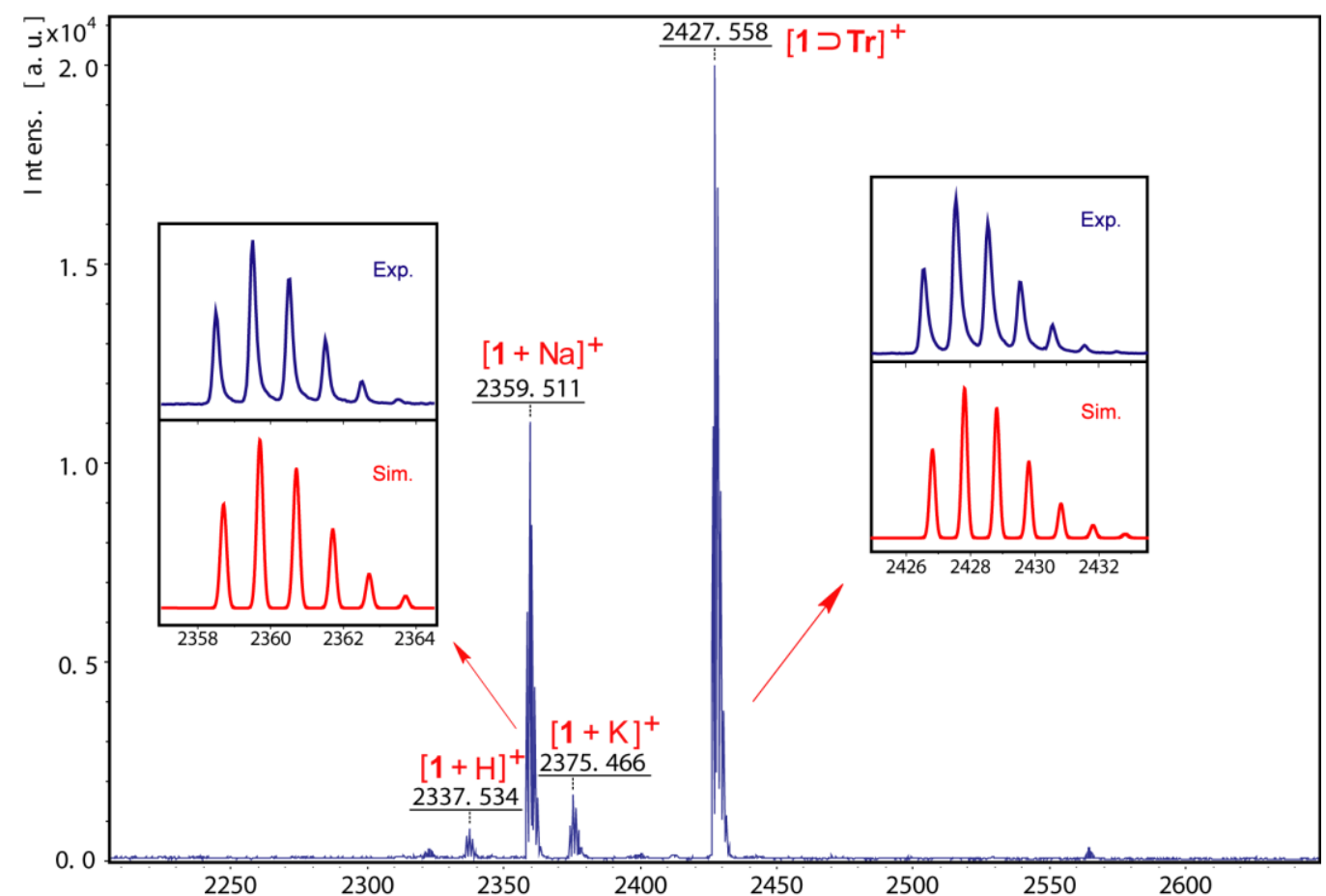

Figure S6. MALDI-TOF spectrum of an equimolar solution $\left(\mathrm{CHCl}_{3} / \mathrm{CH}_{3} \mathrm{CN}, 1 / 1\right.$, v/v) of $\mathbf{1}$ and $\mathbf{T r}^{+}$, showing the presence of 1:1 charge-transfer complex [inset: 
experimental isotope distribution (blue) and computer simulation (red)].

\subsection{Job plot for $1 \supset \operatorname{Tr}^{+}$}

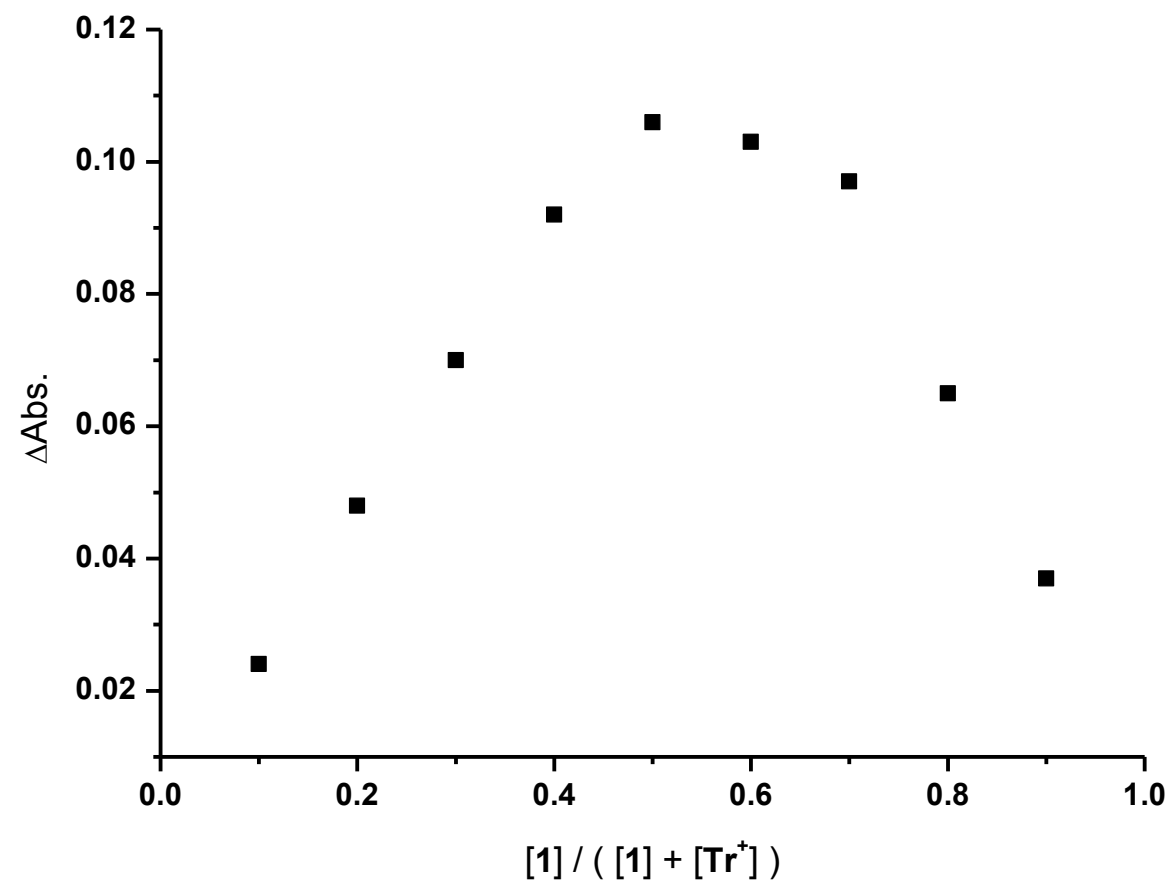

Figure S7. Job plot for the complexation of $\mathbf{1}$ and $\mathbf{T r}^{+}$in $\mathrm{CHCl}_{3} / \mathrm{CH}_{3} \mathrm{CN}(1 / 1, \mathrm{v} / \mathrm{v})$ based on the absorbance at $370 \mathrm{~nm}$, indicating a 1:1 stoichiometry. The total concentration is $2.5 \times 10^{-4} \mathrm{M}$. 


\subsection{FT-IR spectra}

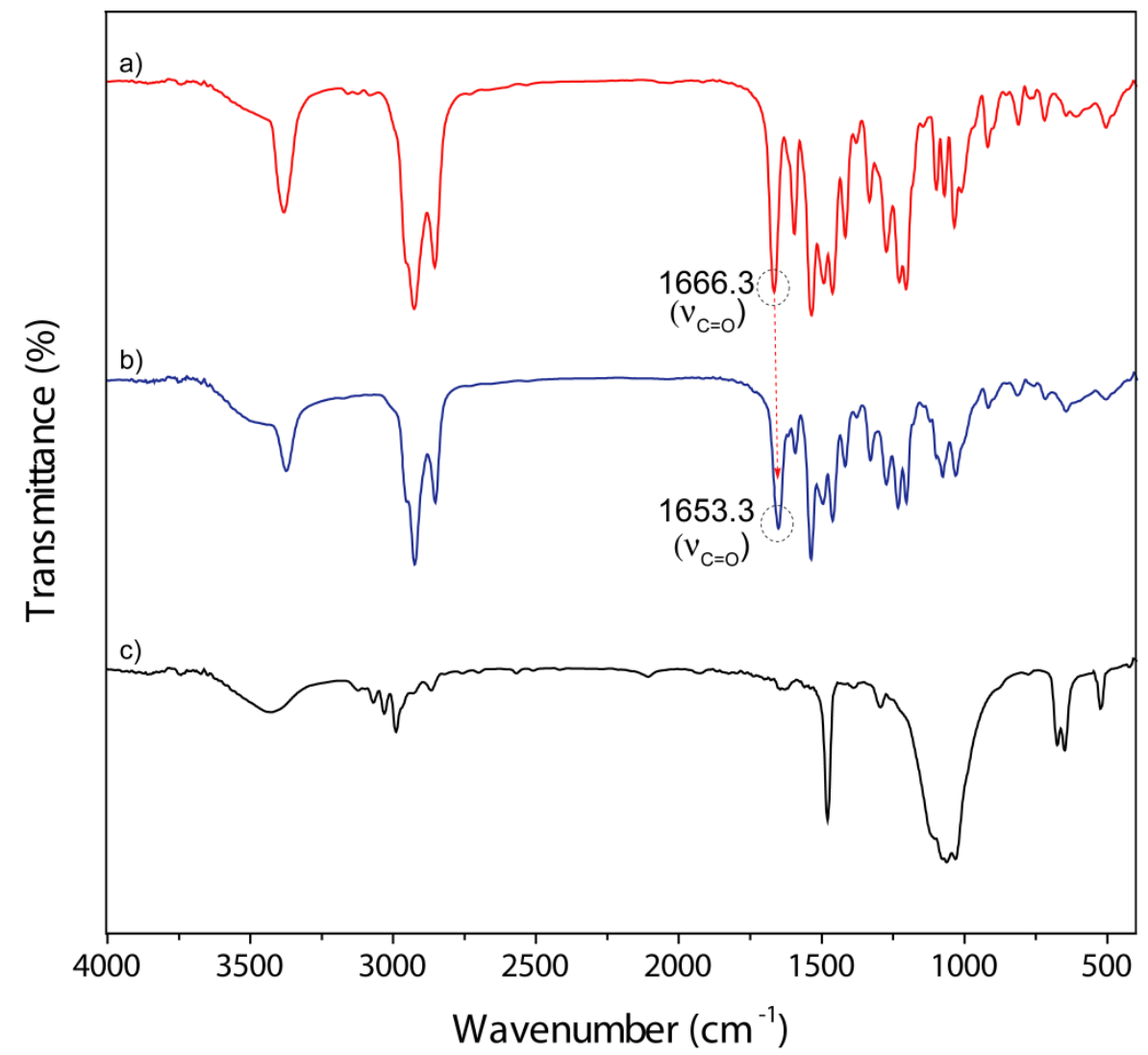

Figure S8. FT-IR spectra of a) iso-C $\mathrm{C}_{16}$-cyclo[6] aramide 1, b) complex $\mathbf{1} \supset \mathbf{T r}^{+}$and c) $\operatorname{Tr}^{+}$.

\subsection{Concentration-dependent ${ }^{1} \mathrm{H}$ NMR spectra of $1 \supset \operatorname{Tr}^{+}$}




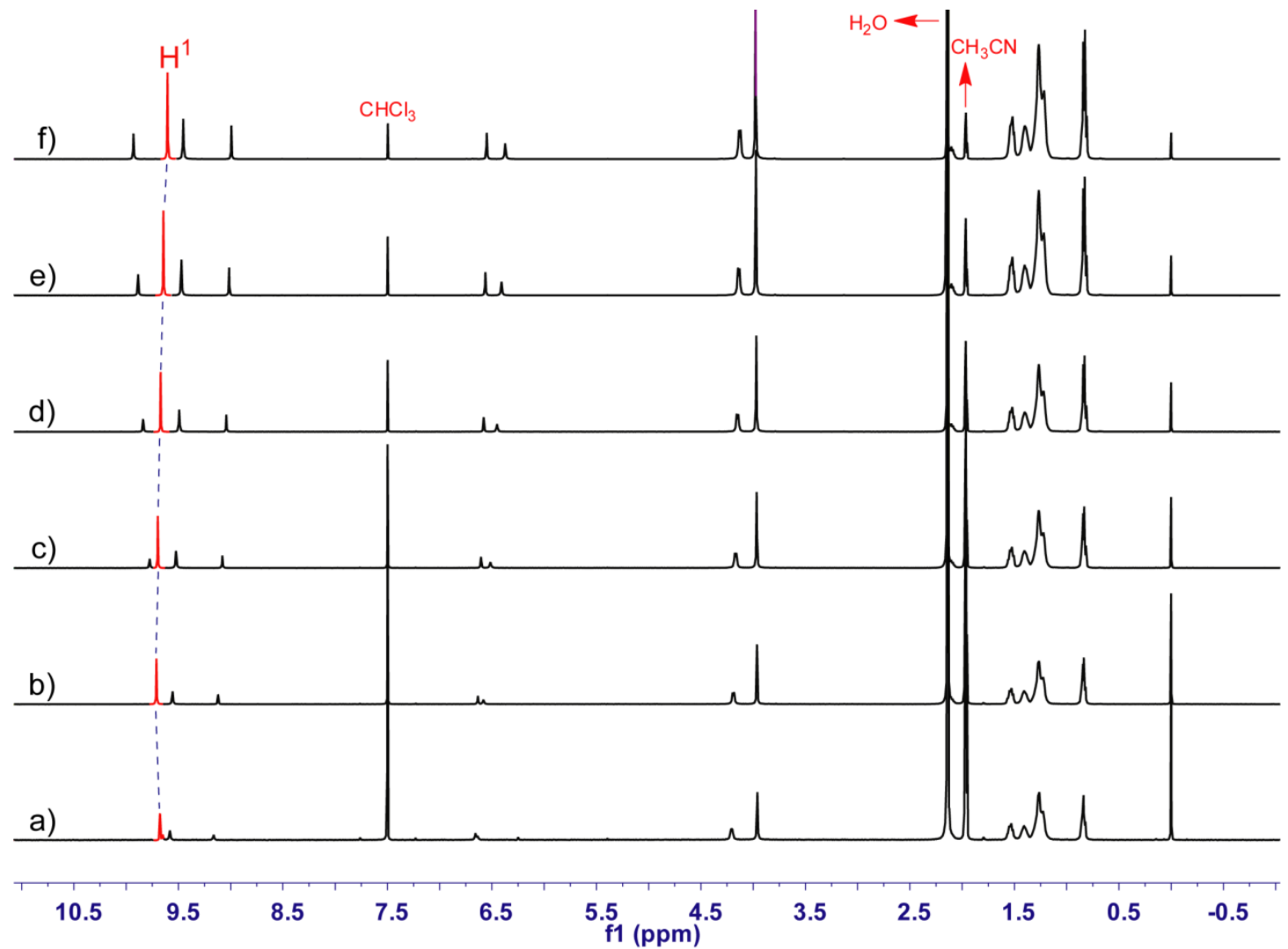

Figure S9. Stacked ${ }^{1} \mathrm{H}$ NMR spectra of $\mathbf{1} \supset \mathbf{T r}^{+}$with various concentrations: a) 0.4 $\mathrm{mM}$, b) $0.8 \mathrm{mM}$, c) $1.6 \mathrm{mM}$, d) $3.2 \mathrm{mM}$, e) $5.6 \mathrm{mM}$, f) $10 \mathrm{mM}$.

\subsection{UV-vis titration of $1 \supset \operatorname{Tr}^{+}$}

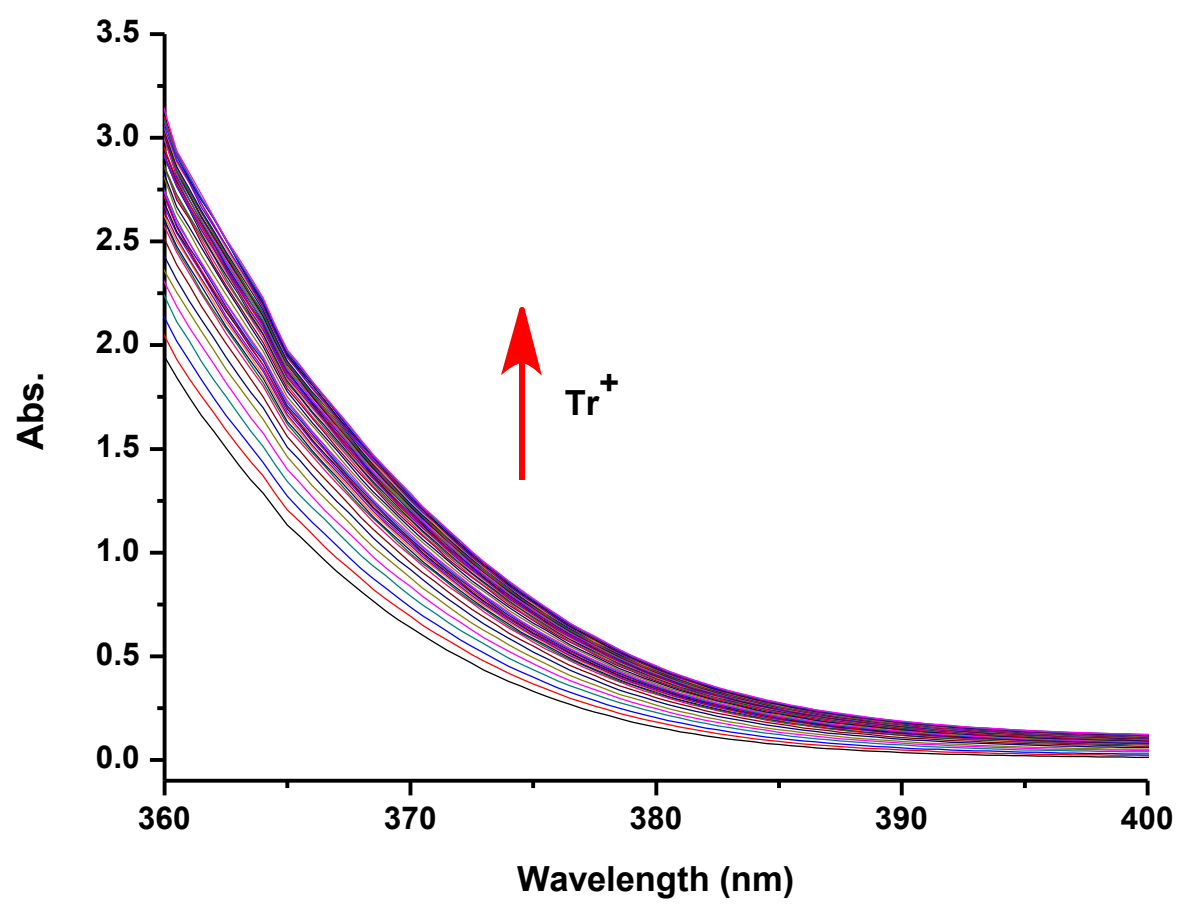

Figure S10. UV-vis spectra of 1 upon addition of $\mathbf{T r}^{+}\left(0-5.0\right.$ equiv) in $\mathrm{CHCl}_{3} / \mathrm{CH}_{3} \mathrm{CN}$ 
$(1 / 1, v / v)$ at $298 \mathrm{~K}$.

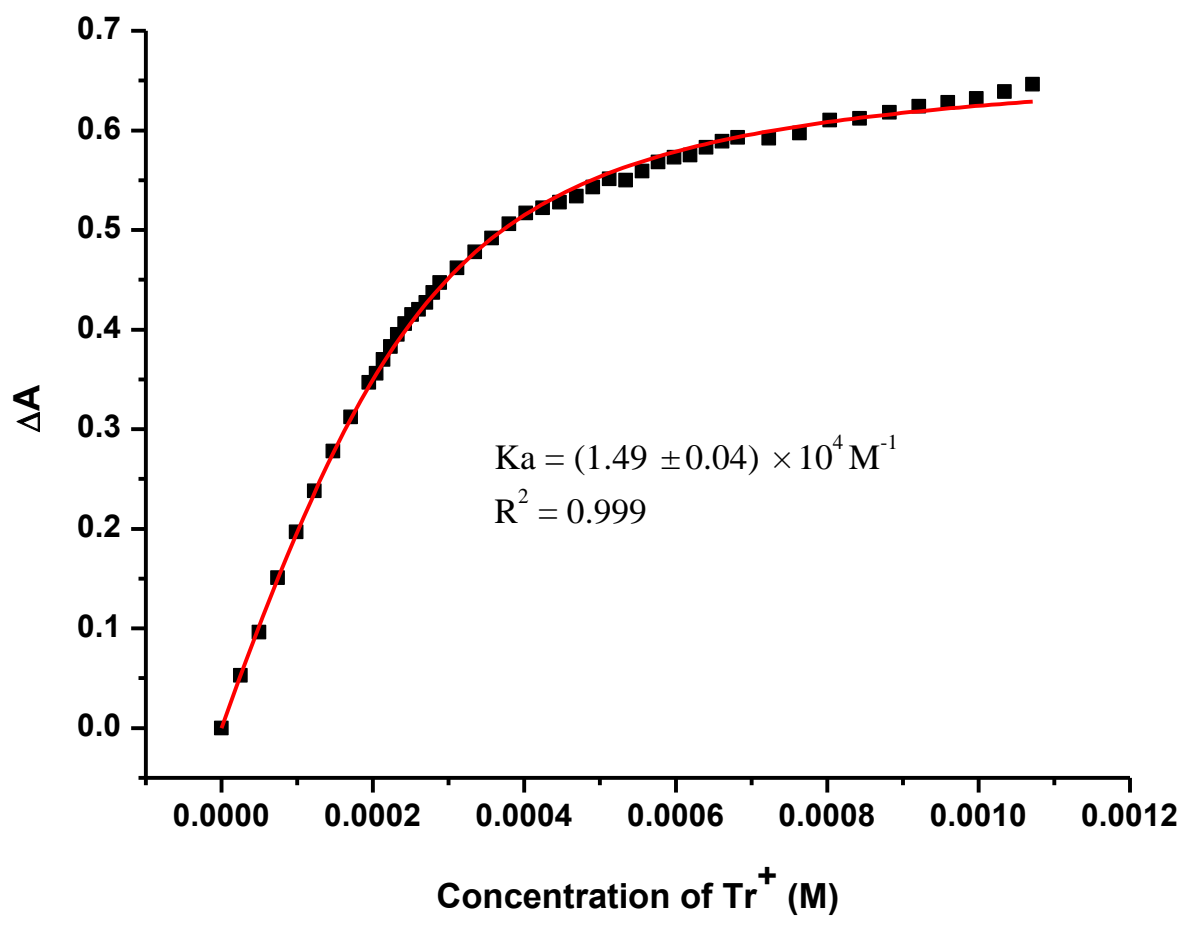

Figure S11. Determination of the binding constant of $\mathbf{1}\left(2.5 \times 10^{-4} \mathrm{M}\right)$ and $\mathbf{T r}^{+}$in $\mathrm{CHCl}_{3} / \mathrm{CH}_{3} \mathrm{CN}(1 / 1, \mathrm{v} / \mathrm{v})$ at $298 \mathrm{~K}$. Fitting result based on the absorbance at $370 \mathrm{~nm}$.

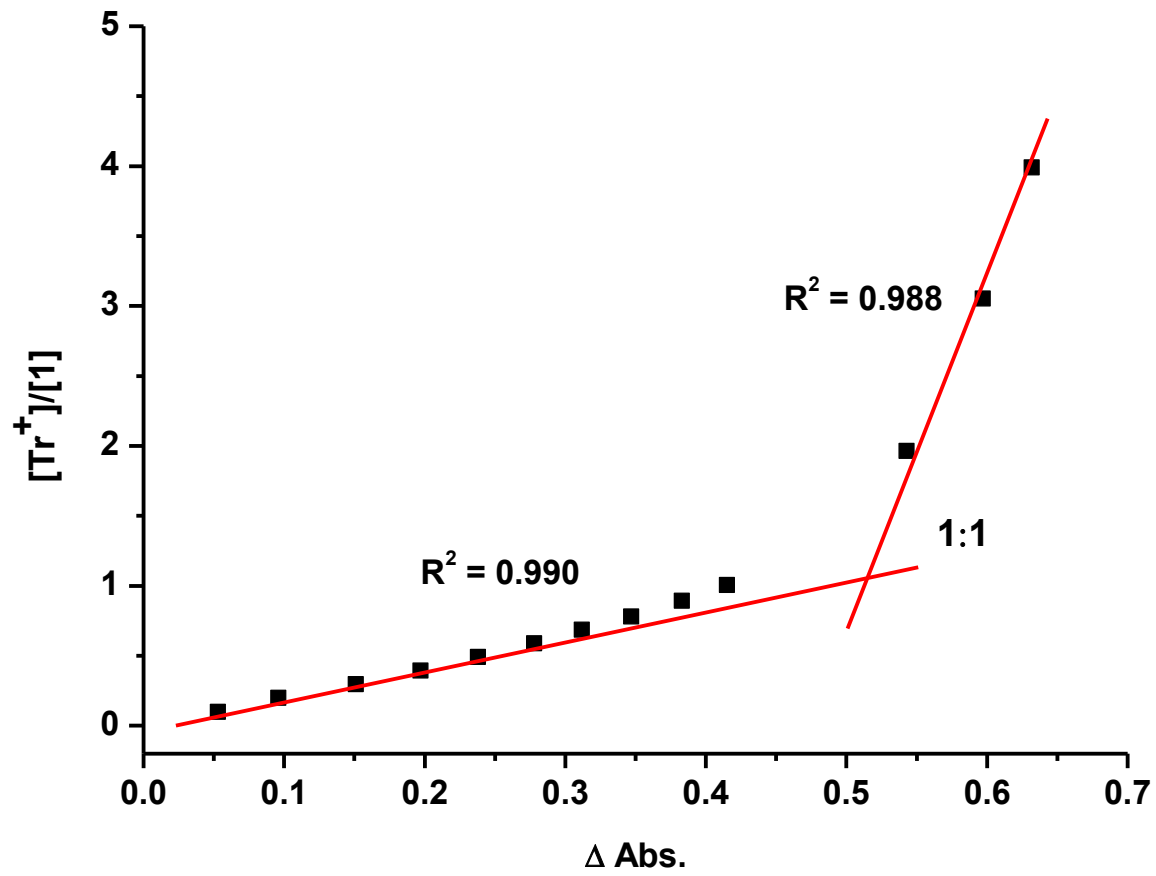

Figure S12. Mole ratio plot for the complexation of 1 and $\mathbf{T r}^{+}$in $\mathrm{CHCl}_{3} / \mathrm{CH}_{3} \mathrm{CN}(1 / 1$, $\mathrm{v} / \mathrm{v}$ ) indicating a 1:1 stoichiometry. 


\section{8 ${ }^{1} \mathrm{H}$ MNR titration of $1 \supset \operatorname{Tr}^{+}$}

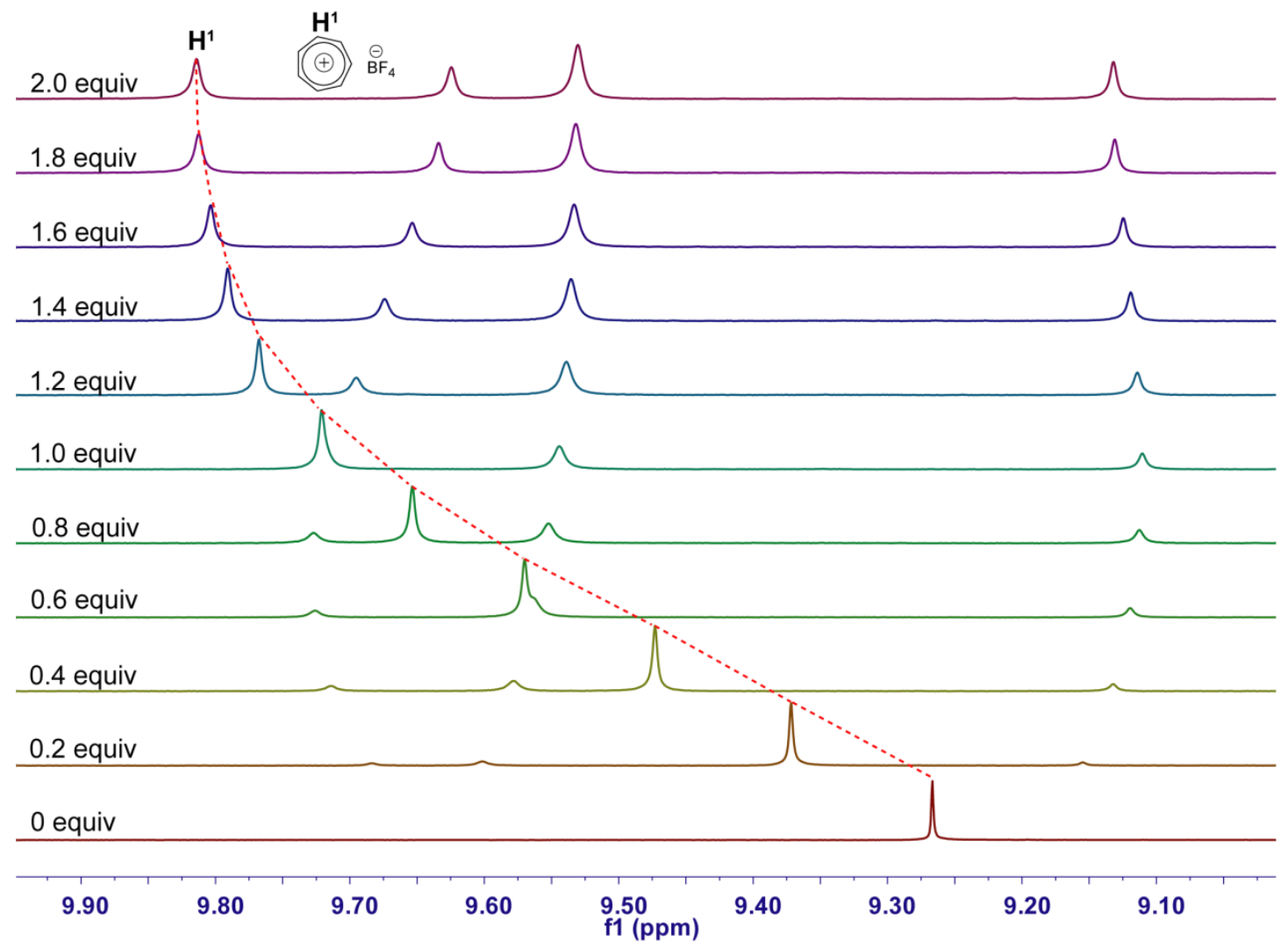

Figure S13. Partial stacked ${ }^{1} \mathrm{H}$ NMR spectra $(400 \mathrm{MHz}, 298 \mathrm{~K})$ of $\mathbf{T r}^{+}(1.0 \mathrm{mM})$ titrated by cyclo[6] aramide 1 (0-2.0 equiv) in $\mathrm{CDCl}_{3} / \mathrm{CD}_{3} \mathrm{CN}(1 / 1, \mathrm{v} / \mathrm{v})$.

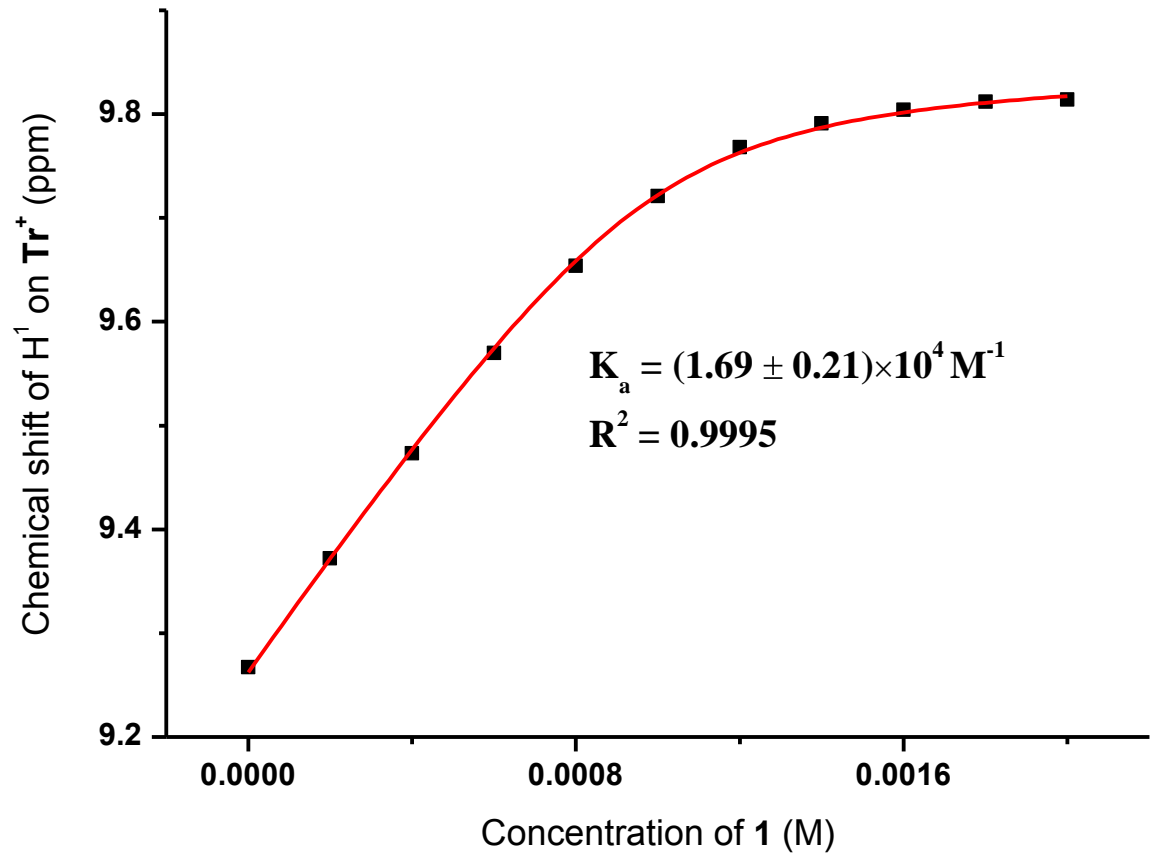

Figure S14. Determination of the binding constant of $\mathbf{1} \supset \mathbf{T r}^{+}$in $\mathrm{CDCl}_{3} / \mathrm{CD}_{3} \mathrm{CN}(1 / 1$, v/v) at $298 \mathrm{~K}$. Fitting result based on $\mathrm{H}^{1}$ of $\mathbf{T r}^{+}$. 


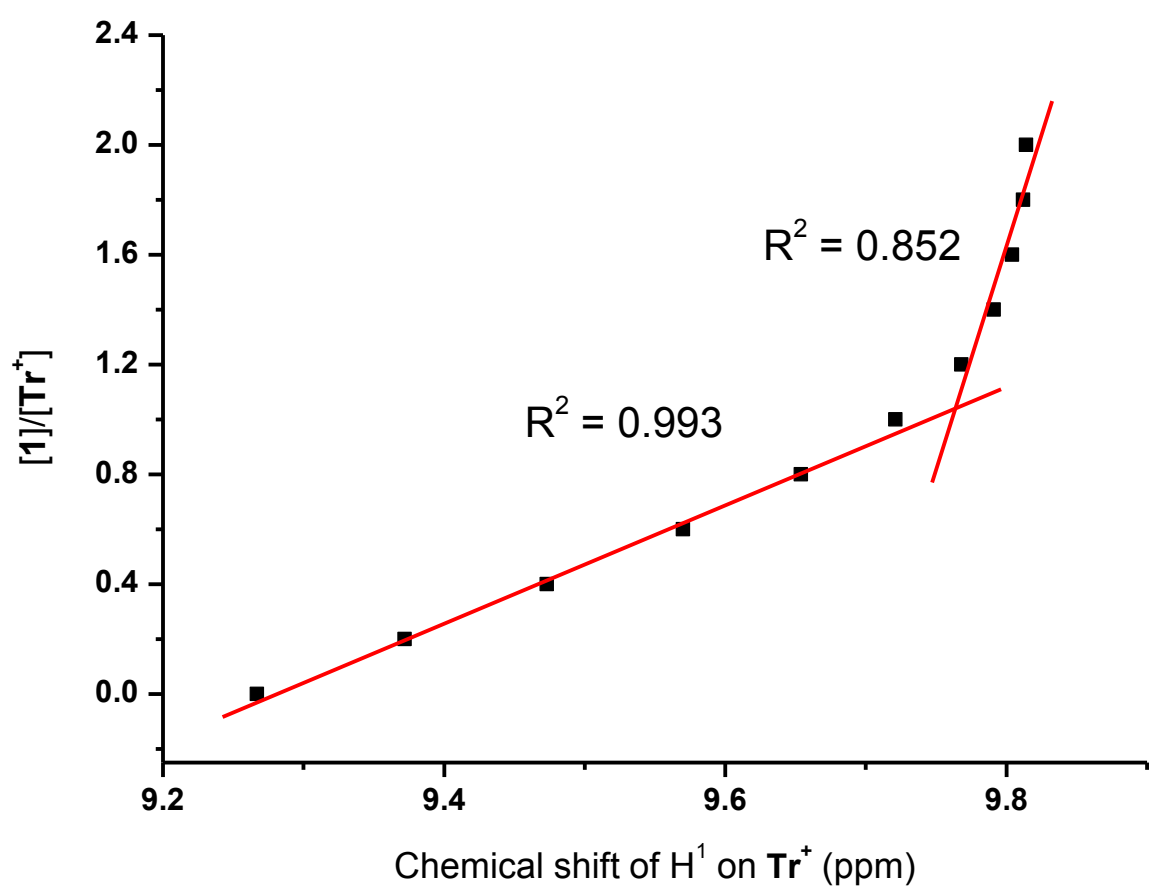

Figure S15. Mole ratio plot for the complexation of $\mathbf{1}$ and $\mathbf{T r}^{+}$in $\mathrm{CDCl}_{3} / \mathrm{CD}_{3} \mathrm{CN}(1 / 1$, $\mathrm{v} / \mathrm{v}$ ) indicating a 1:1 stoichiometry. 


\section{Host-guest complexation of 3 and $T^{\mathrm{r}+}$}

\section{$5.1{ }^{1} \mathrm{H}$ NMR spectra of $3 \supset \operatorname{Tr}^{+}$}

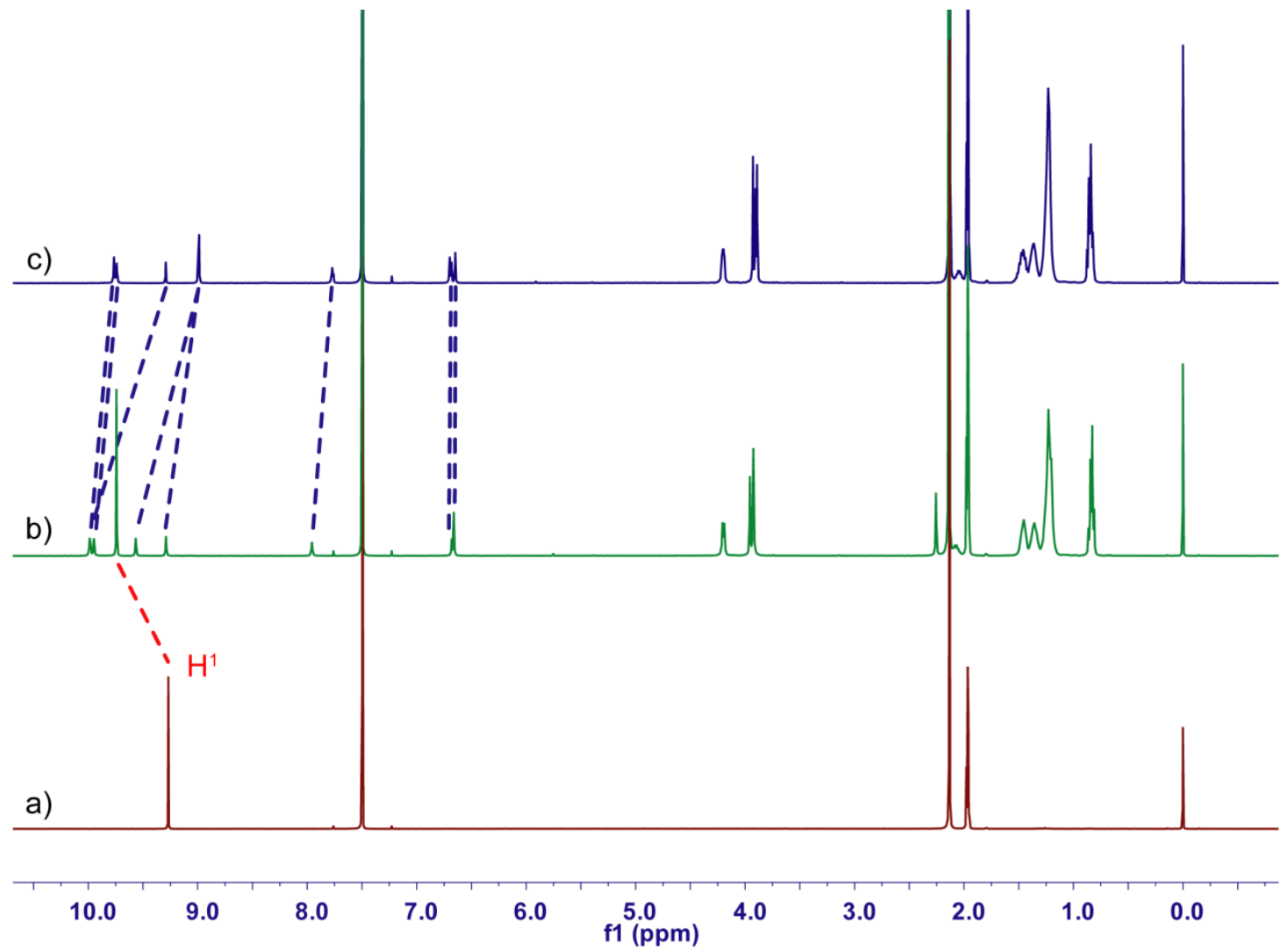

Figure S16. Stacked ${ }^{1} \mathrm{H}$ NMR spectra $\left(400 \mathrm{MHz}, \mathrm{CDCl}_{3} / \mathrm{CD}_{3} \mathrm{CN}, 1 / 1, \mathrm{v} / \mathrm{v}\right)$ of a) $\mathbf{T r}^{+}$ $(2.0 \mathrm{mM})$, b) $\mathbf{3} \supset \mathbf{T r}^{+}(2.0 \mathrm{mM})$, and c) $\mathbf{3}(2.0 \mathrm{mM})$. 


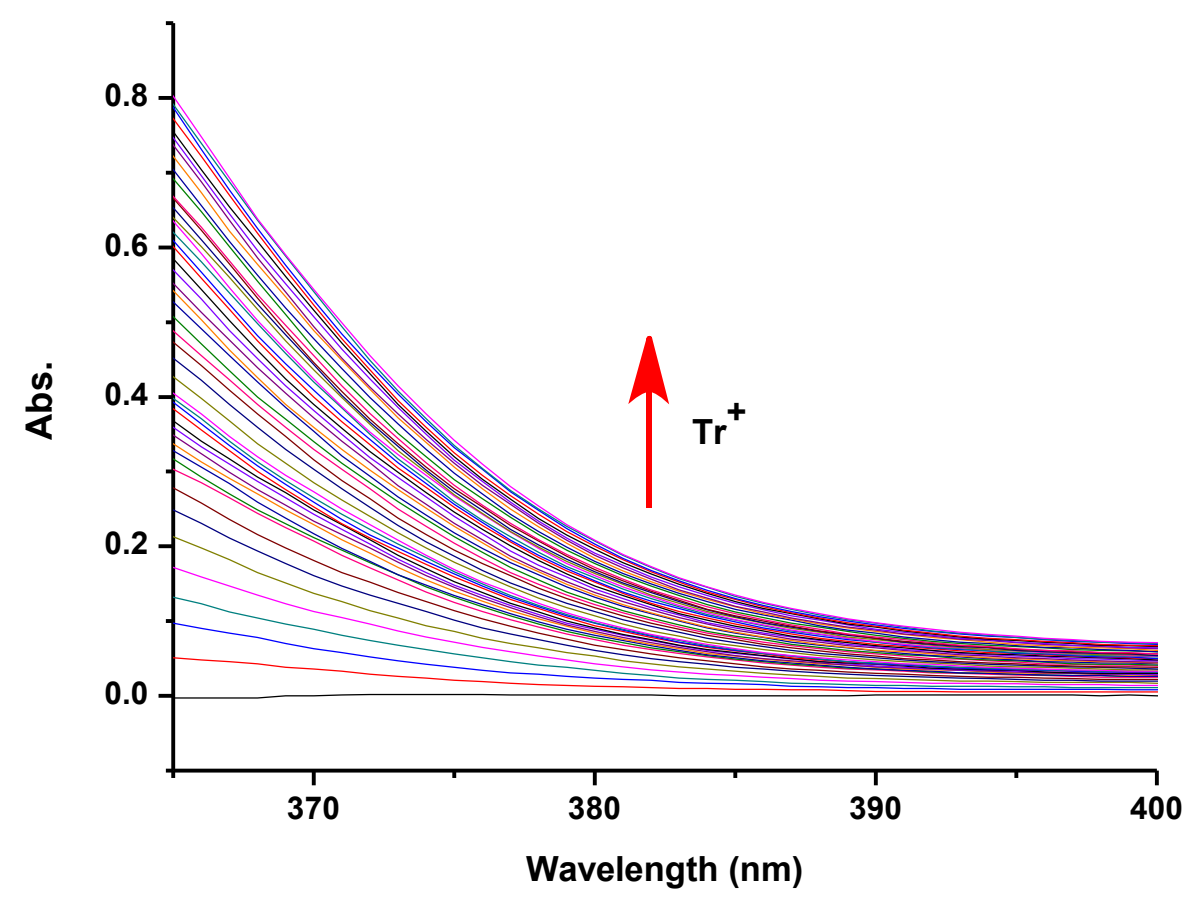

Figure S17. UV-vis spectra of 3 upon addition of $\mathbf{T r}^{+}$(0-5.0 equiv) in $\mathrm{CHCl}_{3} / \mathrm{CH}_{3} \mathrm{CN}$ $(1 / 1, v / v)$ at $298 \mathrm{~K}$.

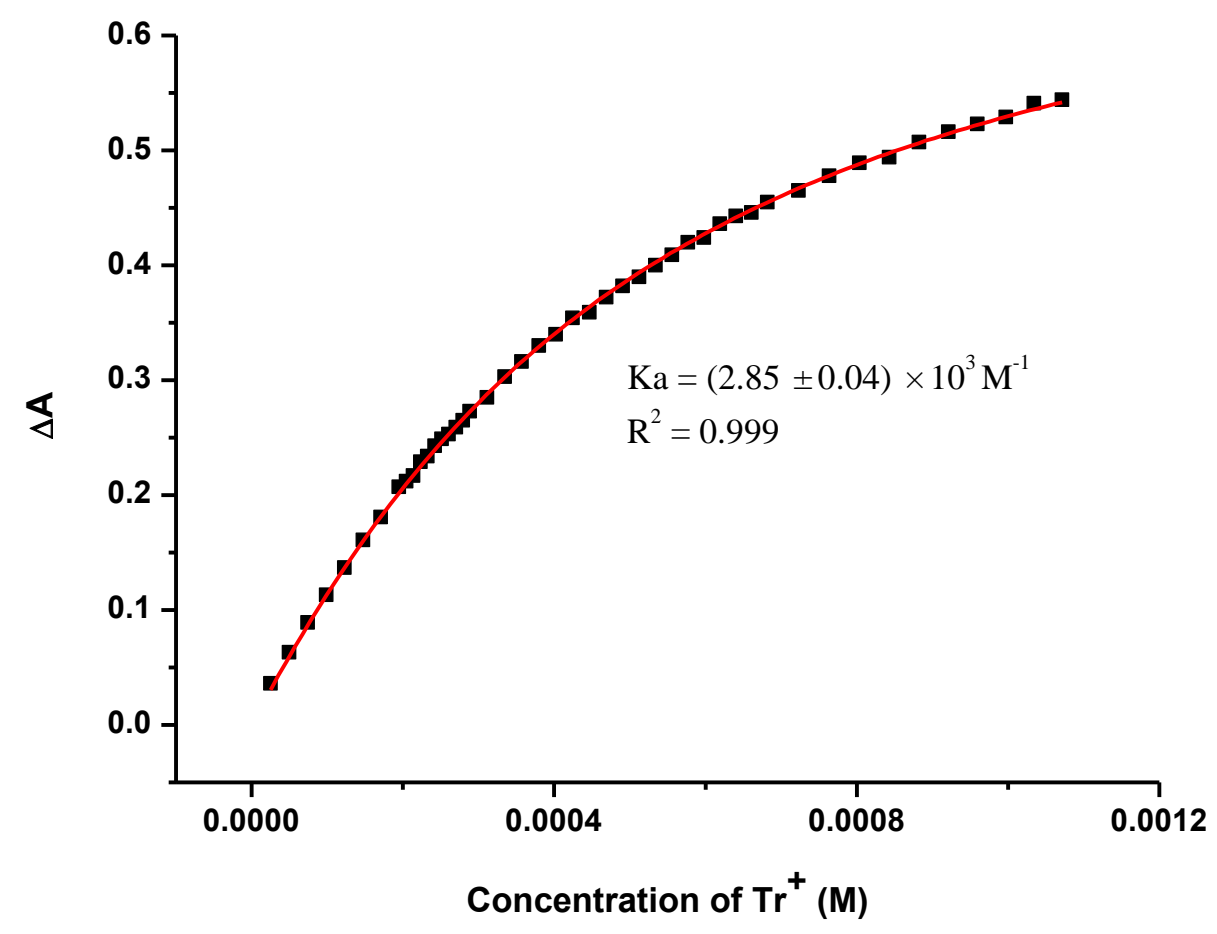

Figure S18. Determination of the binding constant of $\mathbf{3}\left(2.5 \times 10^{-4} \mathrm{M}\right)$ and $\mathbf{T r}^{+}$in 
$\mathrm{CHCl}_{3} / \mathrm{CH}_{3} \mathrm{CN}(1 / 1, \mathrm{v} / \mathrm{v})$ at $298 \mathrm{~K}$. Fitting result based on the absorbance at $370 \mathrm{~nm}$.

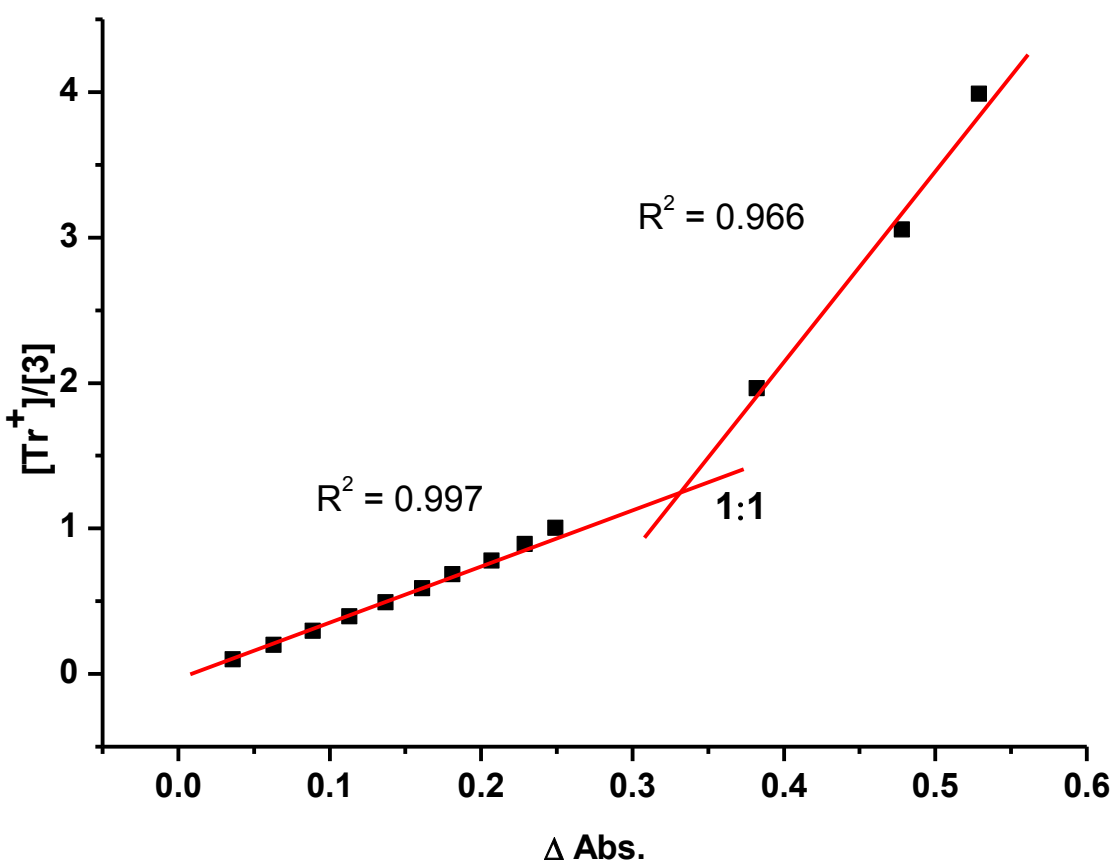

Figure S19. Mole ratio plot for the complexation of $\mathbf{3}$ and $\mathbf{T r}^{+}$in $\mathrm{CHCl}_{3} / \mathrm{CH}_{3} \mathrm{CN}(1 / 1$, $\mathrm{v} / \mathrm{v}$ ) indicating a 1:1 stoichiometry. 


\section{UV-vis spectra of $1 \supset \mathrm{Tr}^{+}$after addition of DBuA}

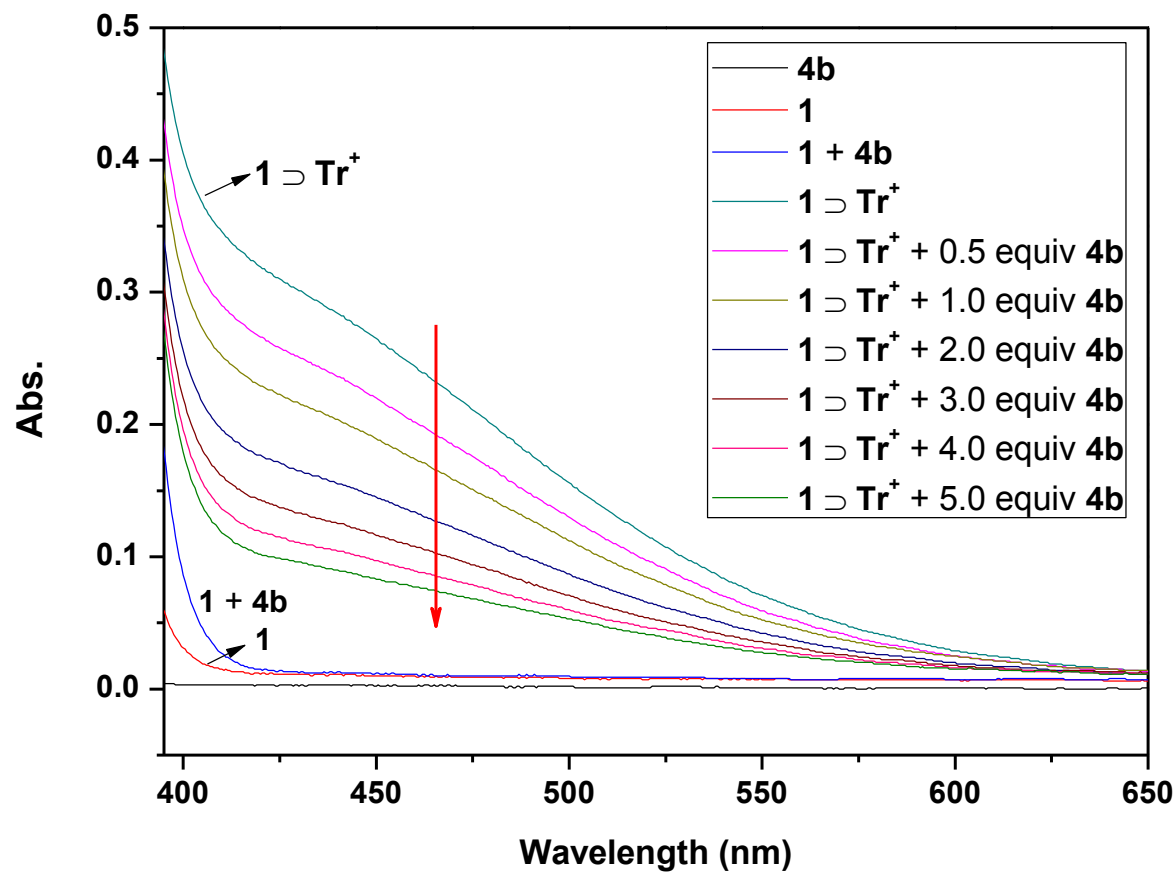

Figure 20. UV-vis spectra of complex $1 \supset \mathbf{T r}^{+}(1 \mathrm{mM})$ and after addition of different equivalents of $\mathbf{4 b}$ in $\mathrm{CHCl}_{3} / \mathrm{CH}_{3} \mathrm{CN}(1 / 1, \mathrm{v} / \mathrm{v})$.

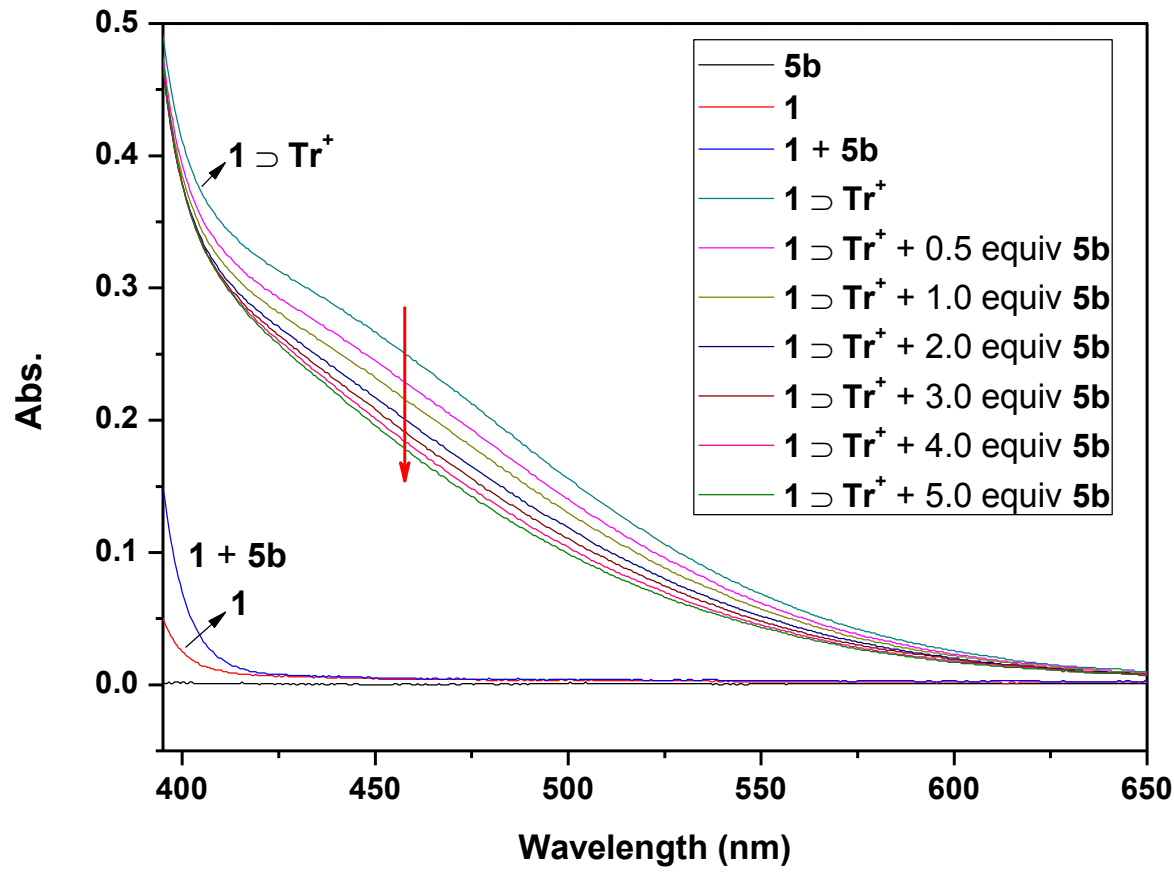

Figure 21. UV-vis spectra of complex $\mathbf{1} \supset \operatorname{Tr}^{+}(1 \mathrm{mM})$ and after addition of different 
equivalents of $\mathbf{5} \mathbf{b}$ in $\mathrm{CHCl}_{3} / \mathrm{CH}_{3} \mathrm{CN}(1 / 1, \mathrm{v} / \mathrm{v})$.

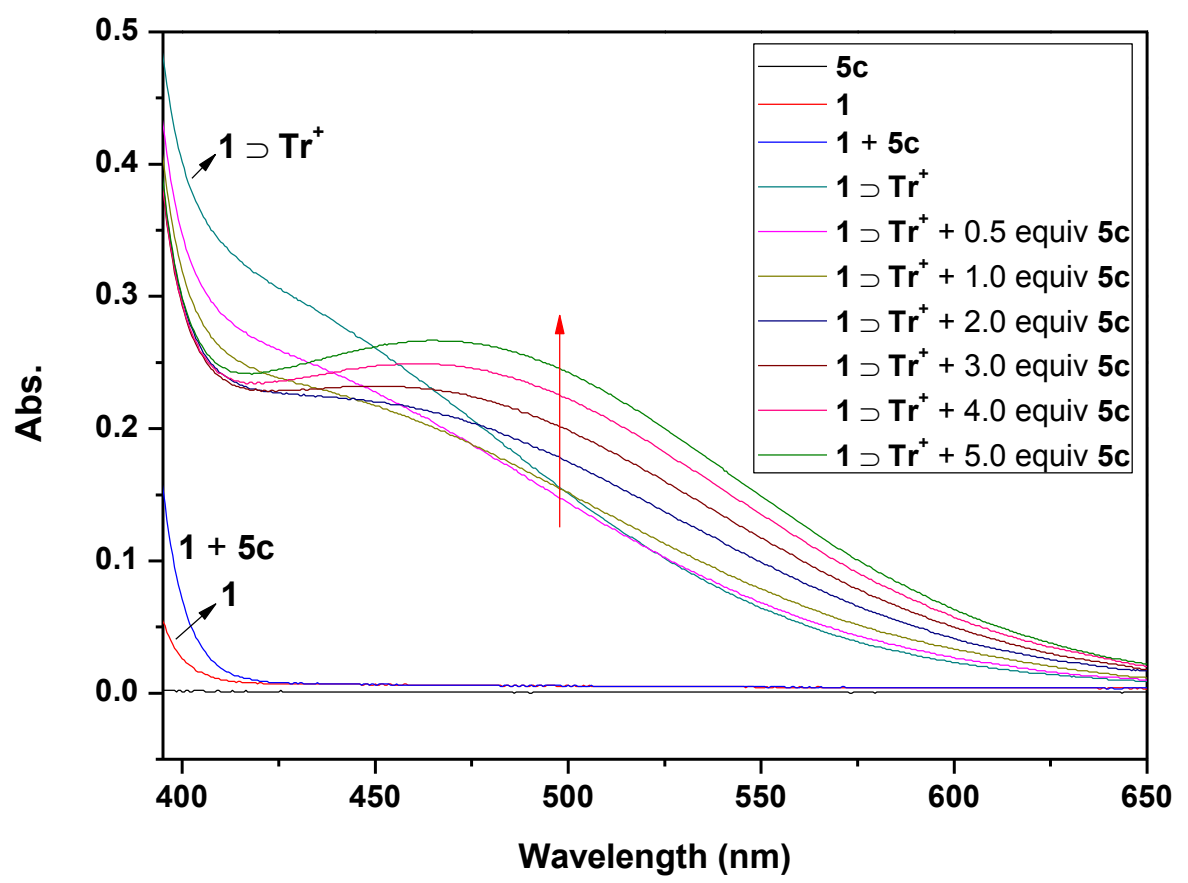

Figure 22. UV-vis spectra of complex $\mathbf{1} \supset \mathbf{T r}^{+}(1 \mathrm{mM})$ and after addition of different equivalents of $\mathbf{5} \mathbf{c}$ in $\mathrm{CHCl}_{3} / \mathrm{CH}_{3} \mathrm{CN}(1 / 1, \mathrm{v} / \mathrm{v})$.

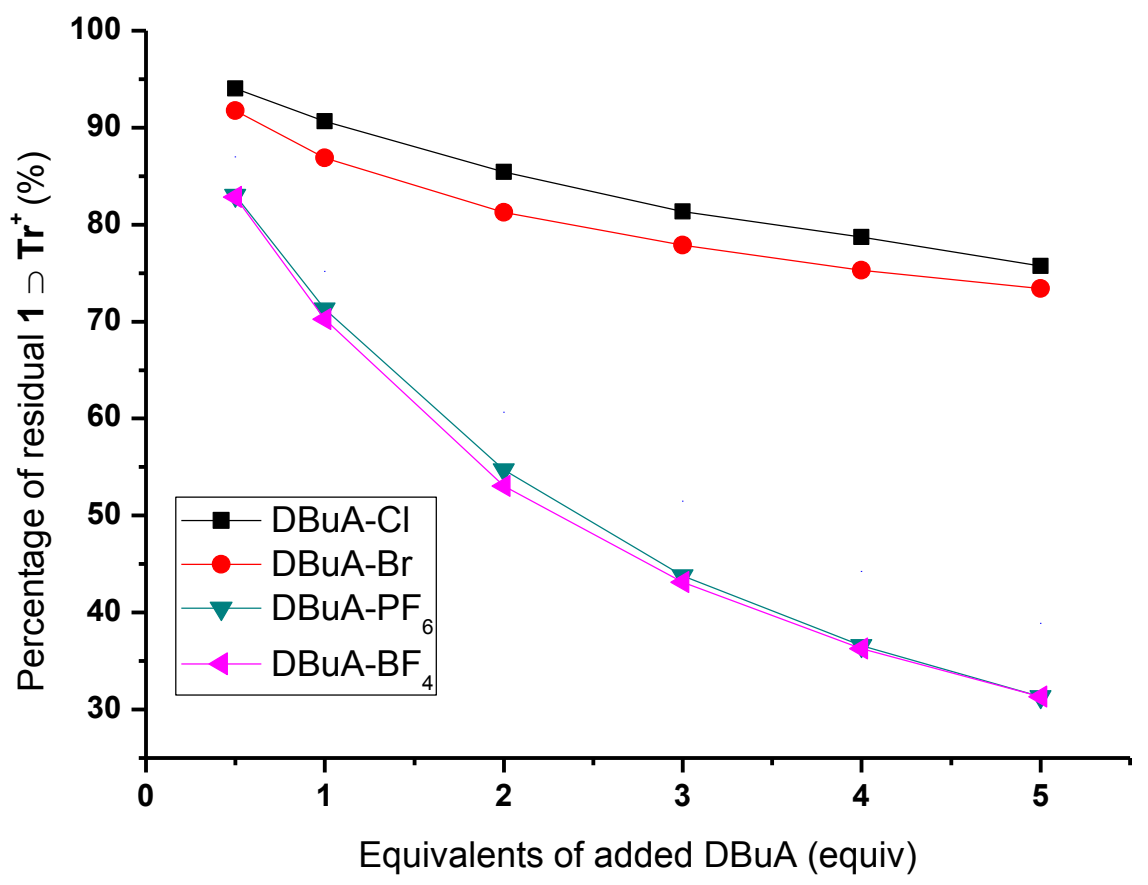

Figure 23. The percentage of residual $1 \supset \operatorname{Tr}^{+}$after addition of various equivalents of DBuA. 


\section{Host-guest complexation of 1 and DBuA}

\subsection{Host-guest complexation of 1 and $4 a$}

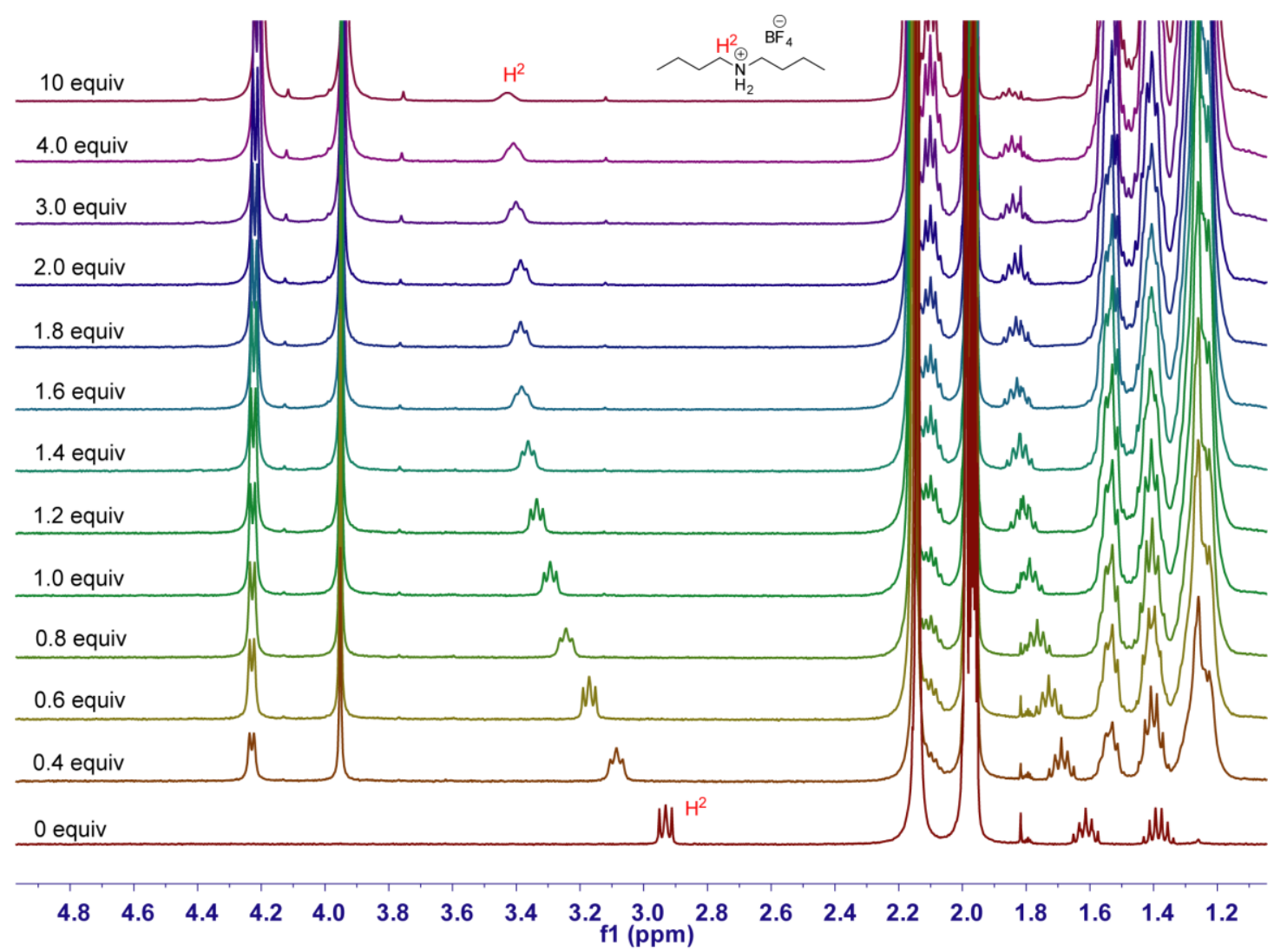

Figure 24. Partial stacked ${ }^{1} \mathrm{H}$ NMR spectra $(400 \mathrm{MHz}, 298 \mathrm{~K})$ of $\mathbf{4 a}(1.0 \mathrm{mM})$ titrated by cyclo[6]aramide $\mathbf{1}$ (0-10 equiv) in $\mathrm{CDCl}_{3} / \mathrm{CD}_{3} \mathrm{CN}(1 / 1$, v/v). 


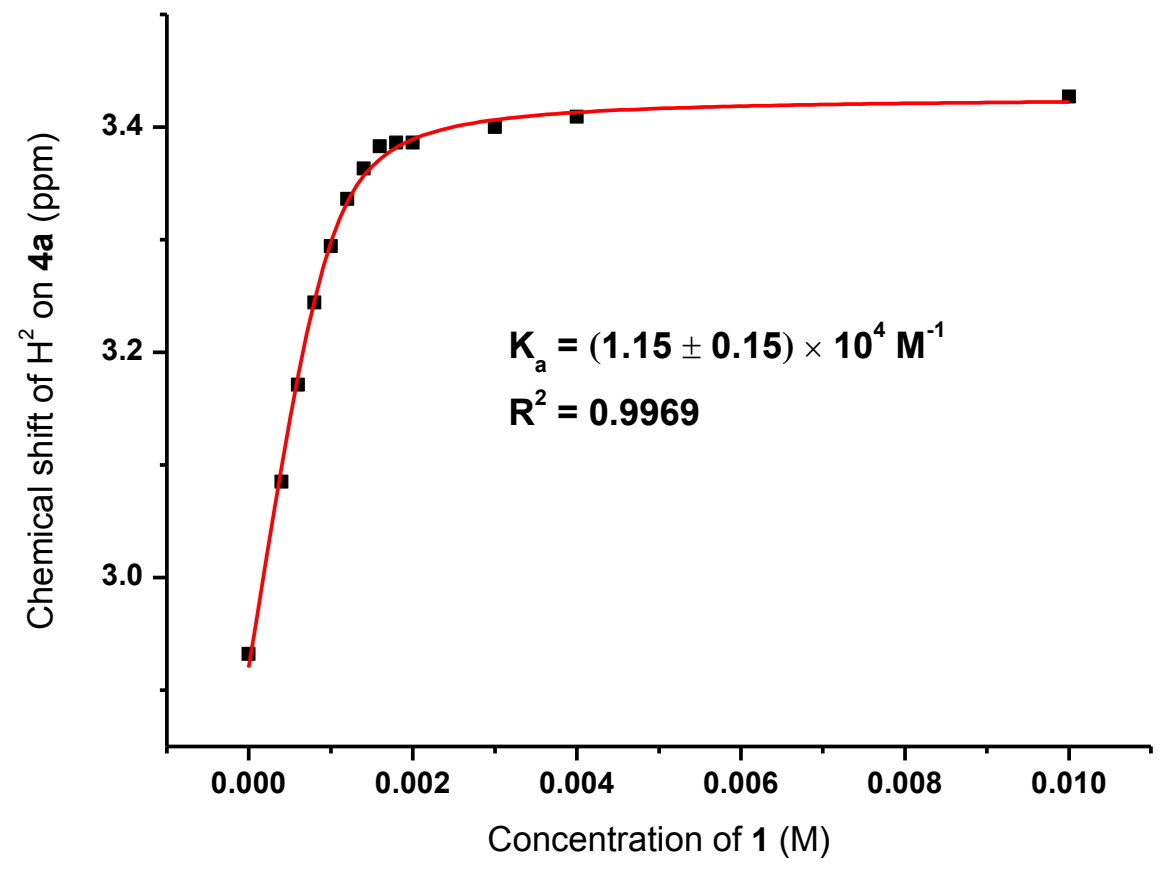

Figure 25. Determination of the binding constant of $\mathbf{1} \supset \mathbf{4 a}$ in $\mathrm{CDCl}_{3} / \mathrm{CD}_{3} \mathrm{CN}(1 / 1$, $\mathrm{v} / \mathrm{v}$ ) at $298 \mathrm{~K}$. Fitting result based on $\mathrm{H}^{2}$ of $\mathbf{4 a}$.

\subsection{Host-guest complexation of 1 and $4 b$}

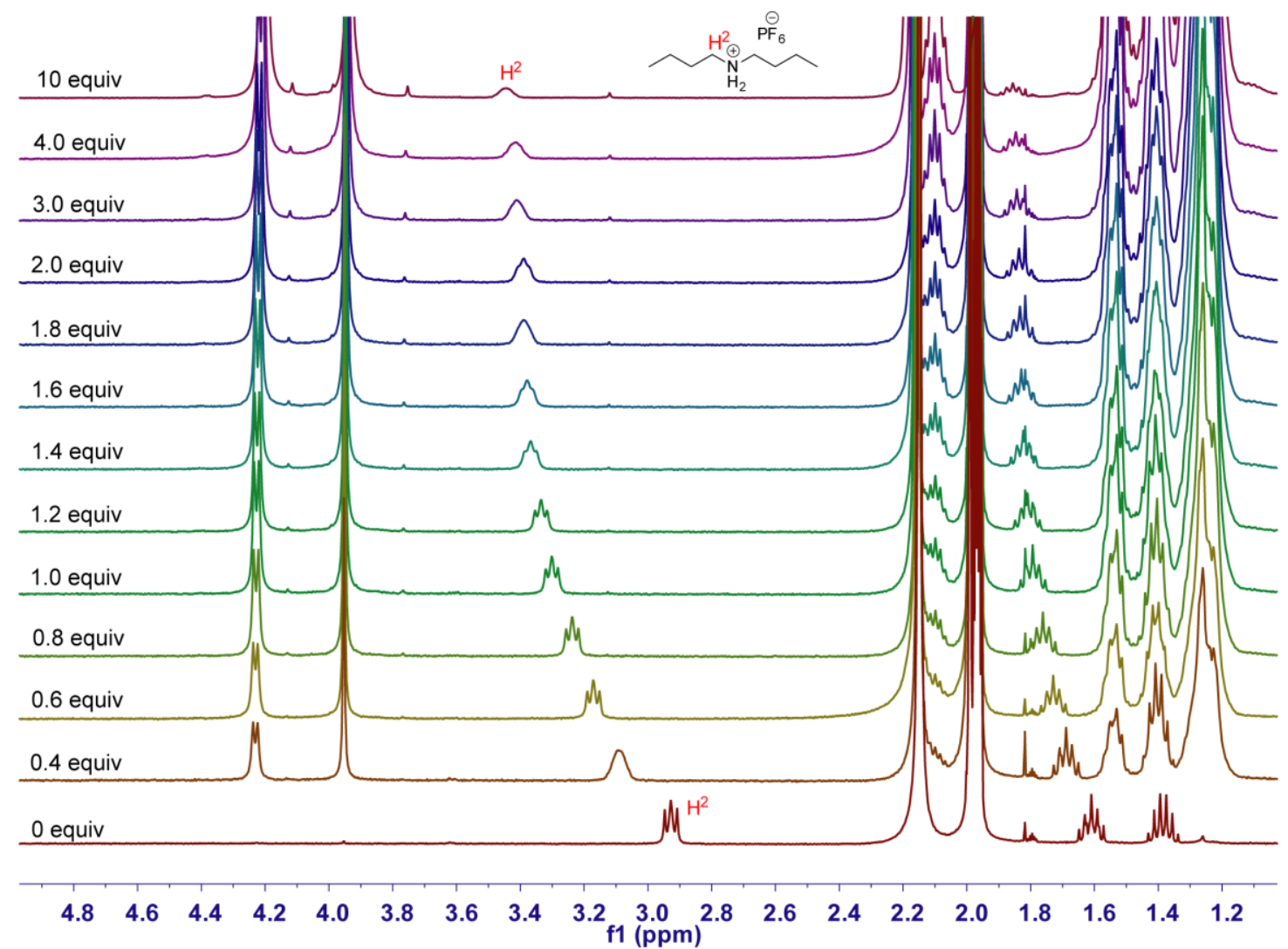

Figure 26. Partial stacked ${ }^{1} \mathrm{H}$ NMR spectra $(400 \mathrm{MHz}, 298 \mathrm{~K})$ of $\mathbf{4 b}(1.0 \mathrm{mM})$ titrated 
by cyclo[6] aramide 1 (0-10 equiv) in $\mathrm{CDCl}_{3} / \mathrm{CD}_{3} \mathrm{CN}(1 / 1, \mathrm{v} / \mathrm{v})$.

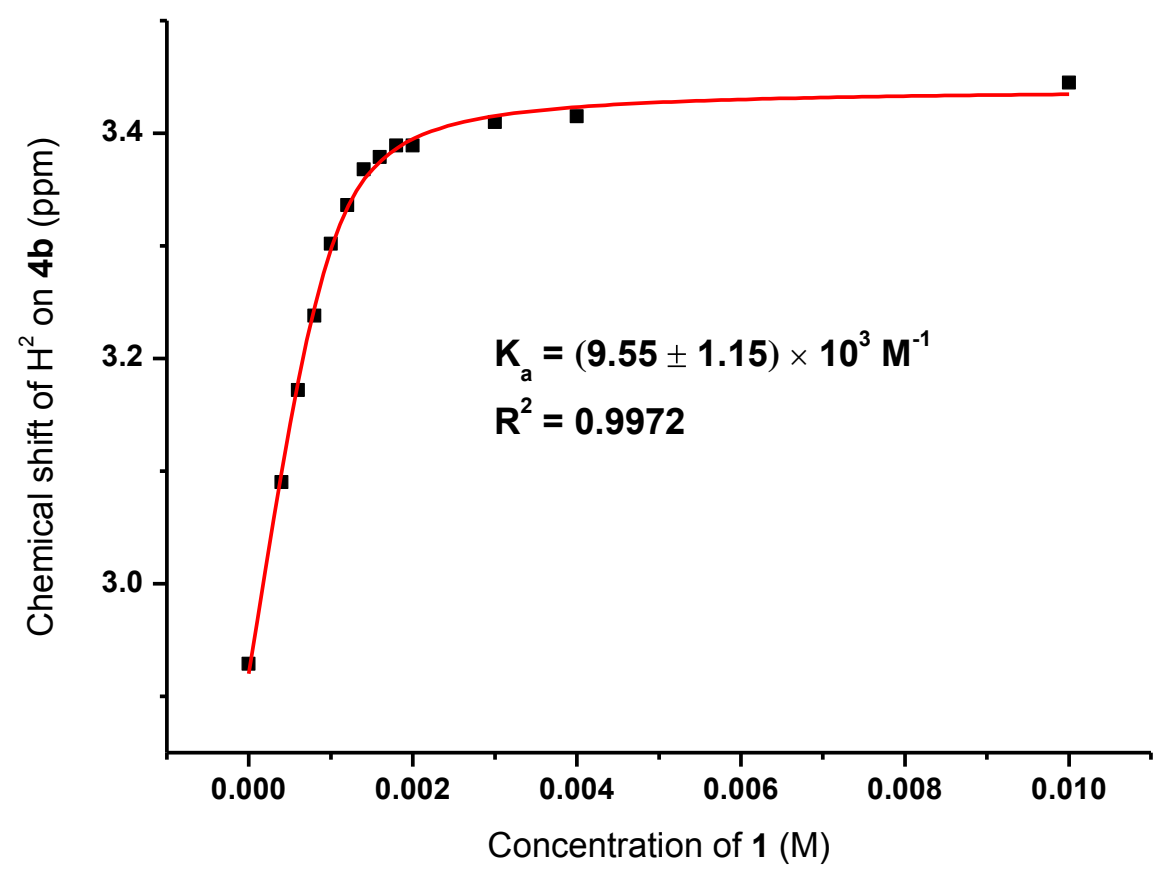

Figure 27. Determination of the binding constant of $\mathbf{1} \supset \mathbf{4 b}$ in $\mathrm{CDCl}_{3} / \mathrm{CD}_{3} \mathrm{CN}(1 / 1$, v/v) at $298 \mathrm{~K}$. Fitting result based on $\mathrm{H}^{2}$ of $\mathbf{4 b}$.

\subsection{Host-guest complexation of 1 and $5 a$}

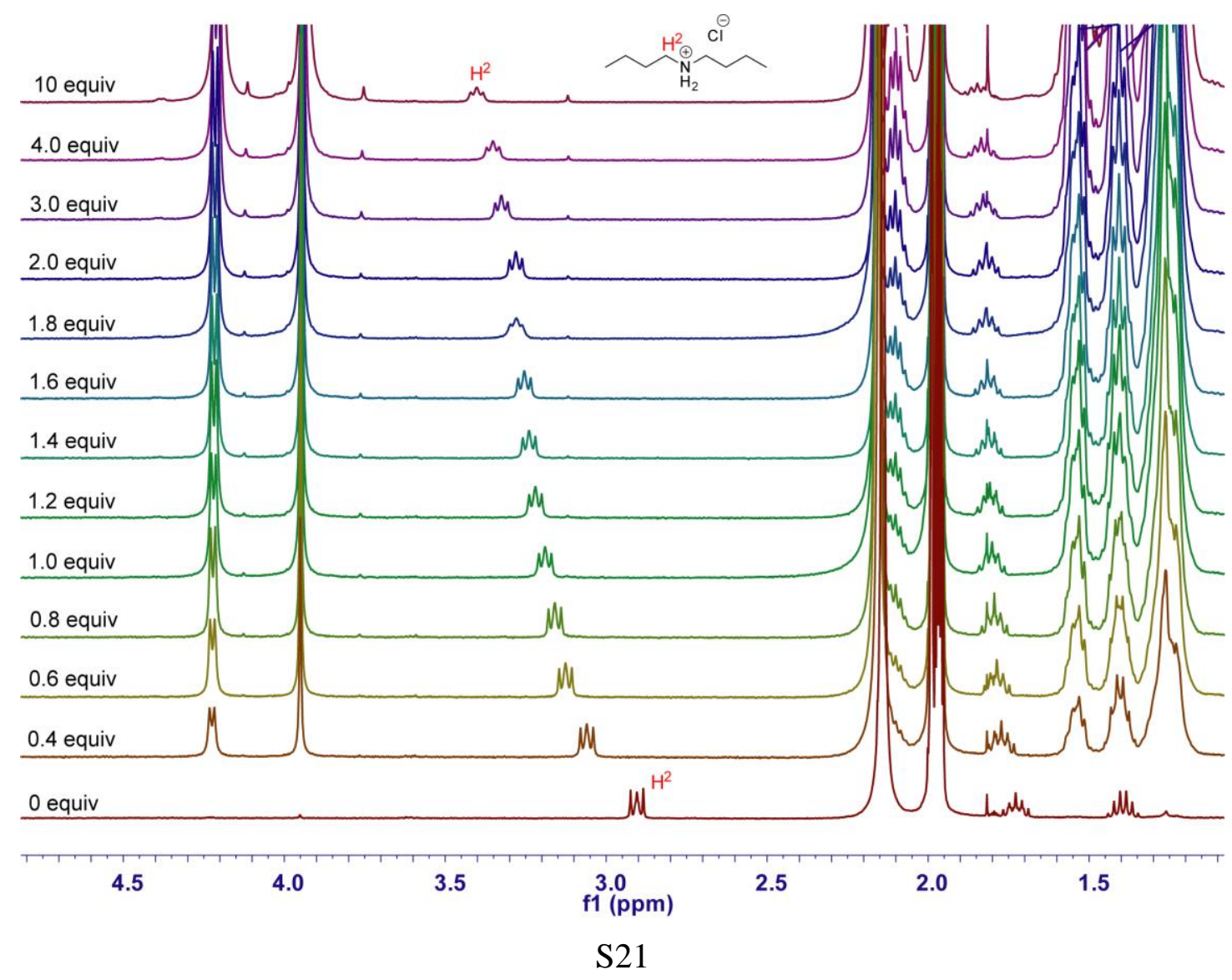


Figure 28. Partial stacked ${ }^{1} \mathrm{H}$ NMR spectra $(400 \mathrm{MHz}, 298 \mathrm{~K})$ of $\mathbf{5 a}(1.0 \mathrm{mM})$ titrated by cyclo[6] aramide $\mathbf{1}$ (0-10 equiv) in $\mathrm{CDCl}_{3} / \mathrm{CD}_{3} \mathrm{CN}(1 / 1$, v/v).

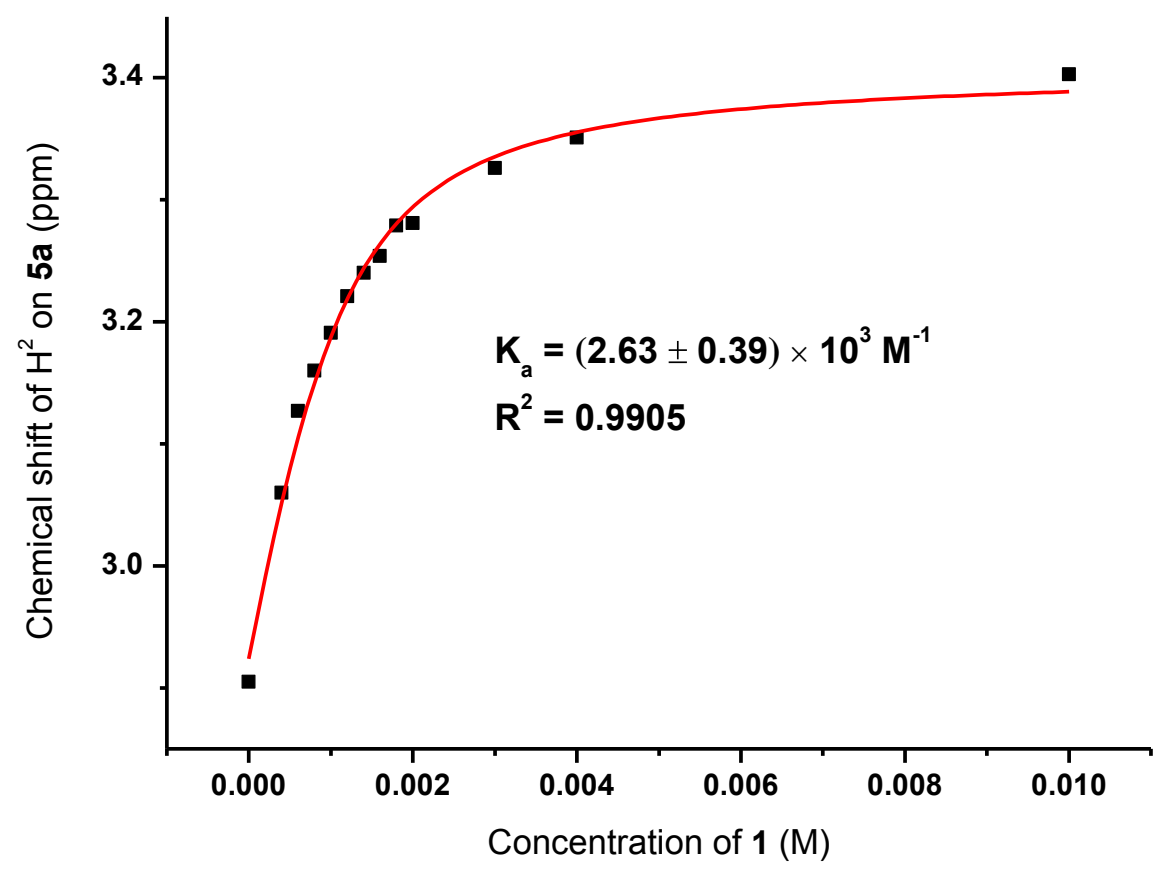

Figure 29. Determination of the binding constant of $\mathbf{1} \supset \mathbf{5 a}$ in $\mathrm{CDCl}_{3} / \mathrm{CD}_{3} \mathrm{CN}(1 / 1$, $\mathrm{v} / \mathrm{v}$ ) at $298 \mathrm{~K}$. Fitting result based on $\mathrm{H}^{2}$ of $\mathbf{5 a}$.

\subsection{Host-guest complexation of 1 and $5 b$}




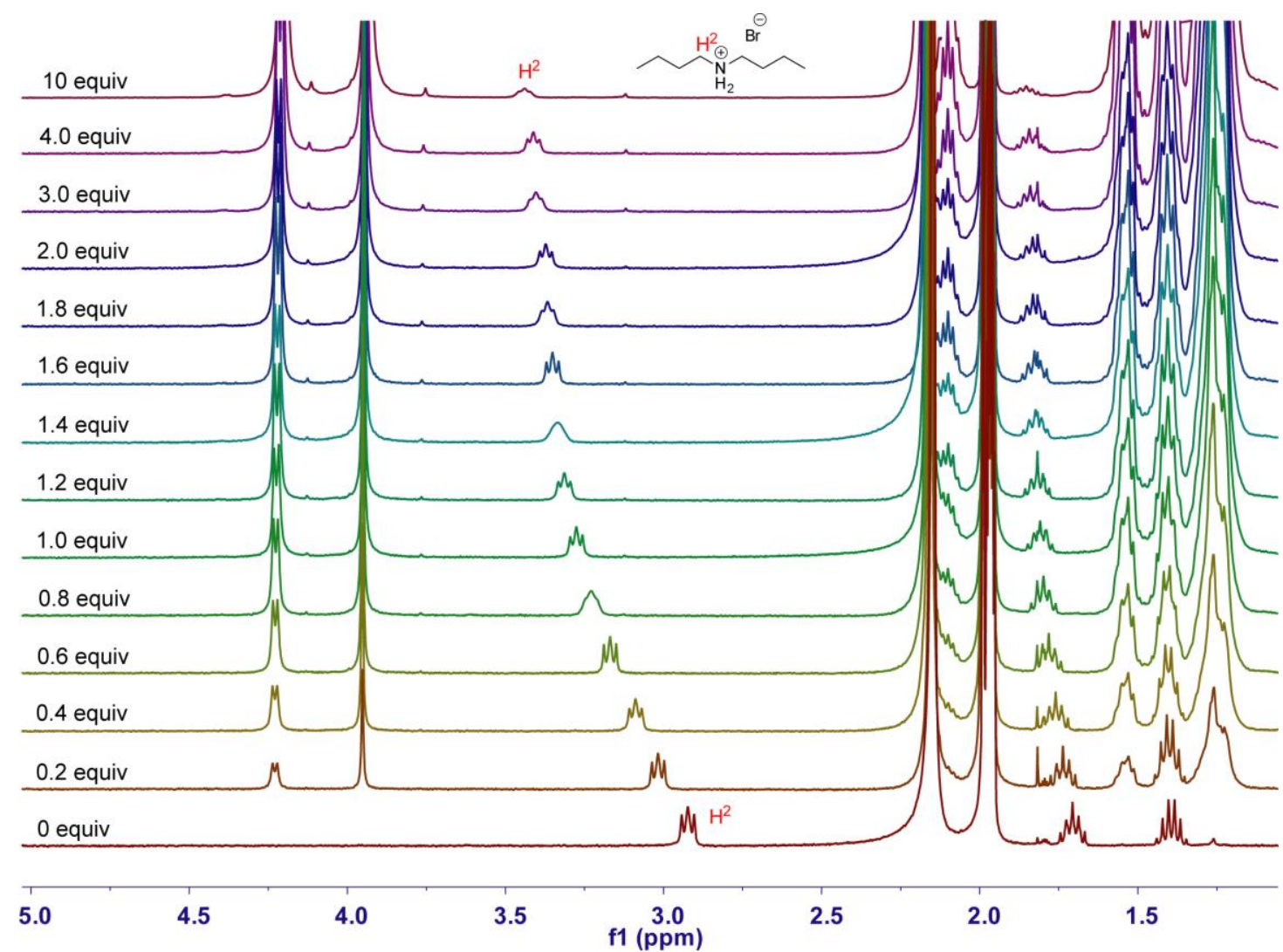

Figure 30. Partial stacked ${ }^{1} \mathrm{H}$ NMR spectra $(400 \mathrm{MHz}, 298 \mathrm{~K})$ of $\mathbf{5 b}(1.0 \mathrm{mM})$ titrated by cyclo[6] aramide 1 (0-10 equiv) in $\mathrm{CDCl}_{3} / \mathrm{CD}_{3} \mathrm{CN}(1 / 1, \mathrm{v} / \mathrm{v})$.

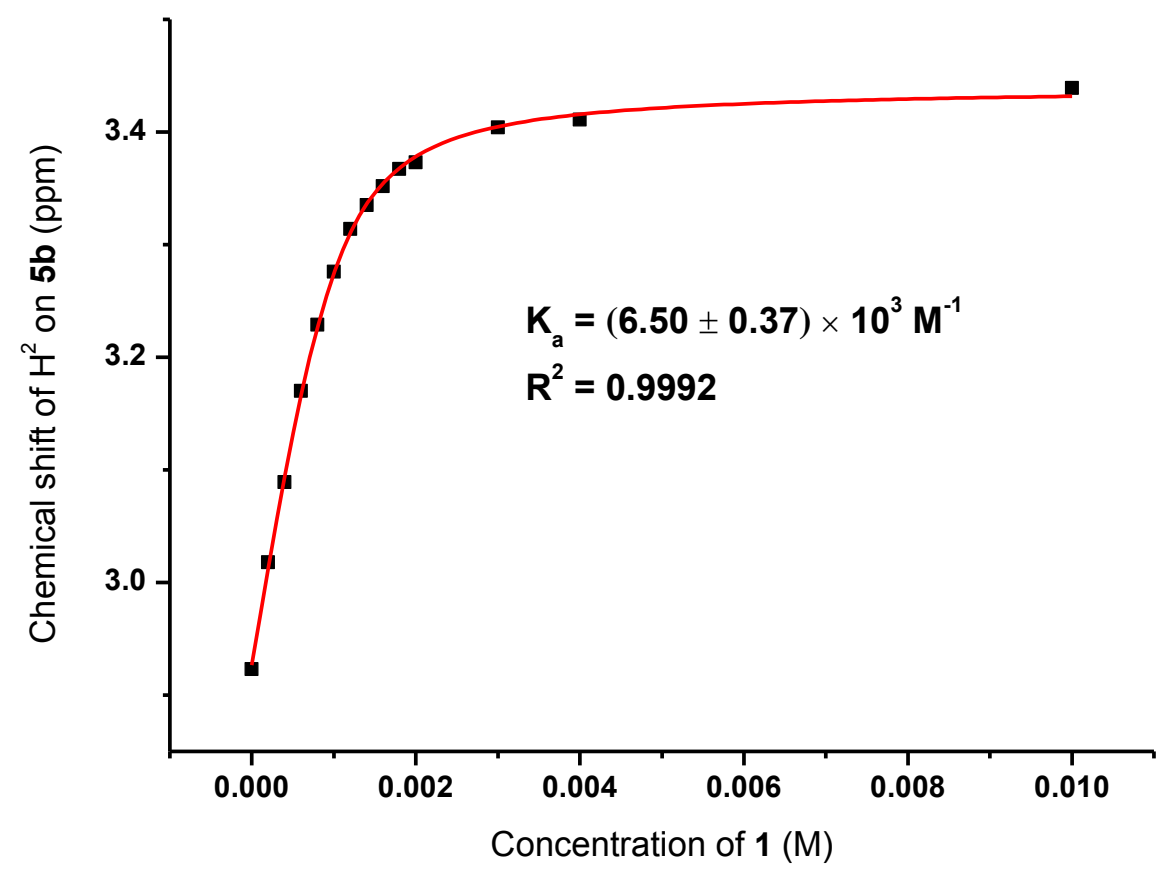

Figure 31. Determination of the binding constant of $\mathbf{1} \supset \mathbf{5 b}$ in $\mathrm{CDCl}_{3} / \mathrm{CD}_{3} \mathrm{CN}(1 / 1$, v/v) at $298 \mathrm{~K}$. Fitting result based on $\mathrm{H}^{2}$ of $\mathbf{5 b}$. 


\subsection{Host-guest complexation of 1 and $5 c$}

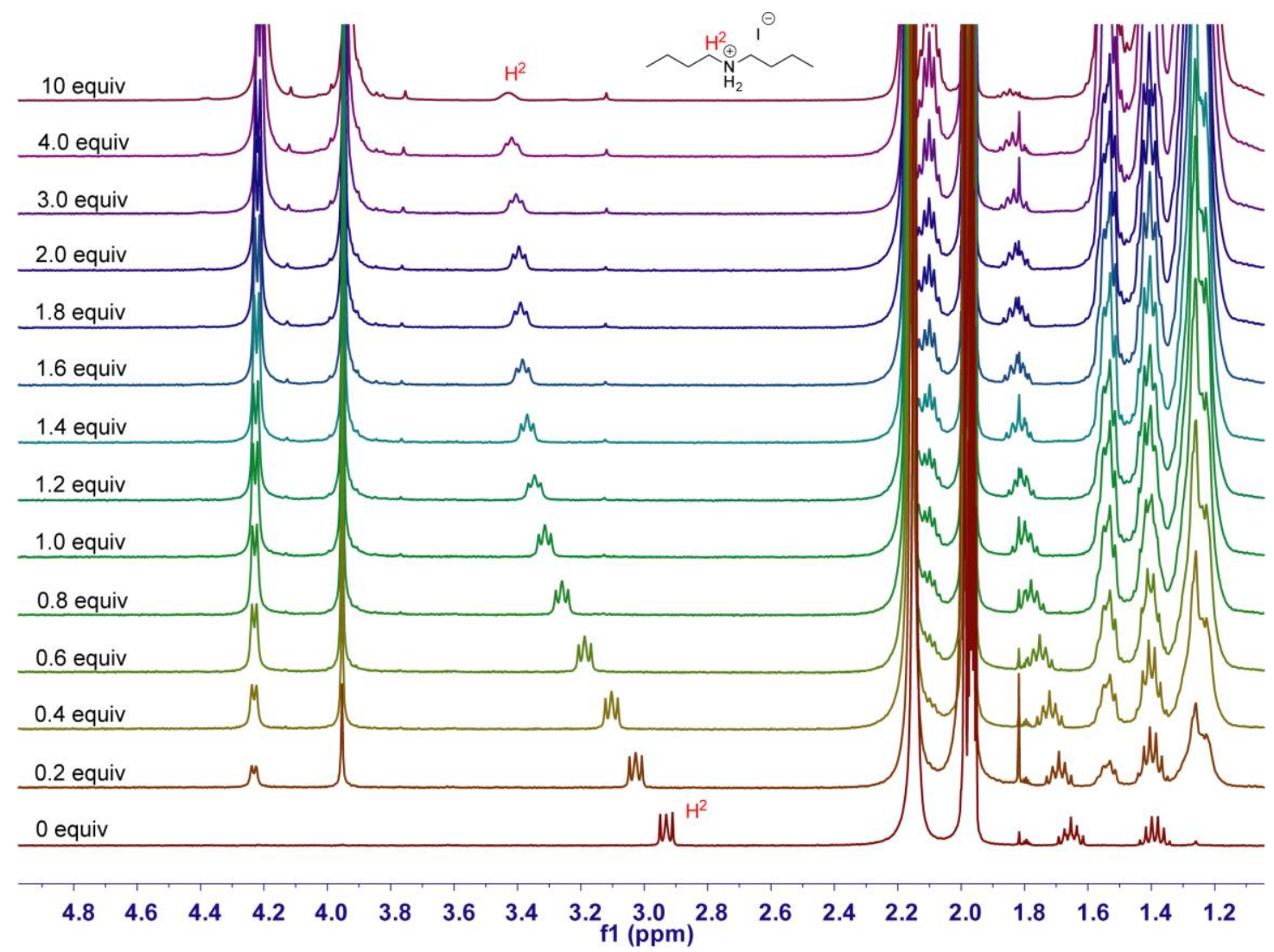

Figure 32. Partial stacked ${ }^{1} \mathrm{H}$ NMR spectra $(400 \mathrm{MHz}, 298 \mathrm{~K})$ of $\mathbf{5 c}(1.0 \mathrm{mM})$ titrated by cyclo[6]aramide 1 (0-10 equiv) in $\mathrm{CDCl}_{3} / \mathrm{CD}_{3} \mathrm{CN}(1 / 1, \mathrm{v} / \mathrm{v})$.

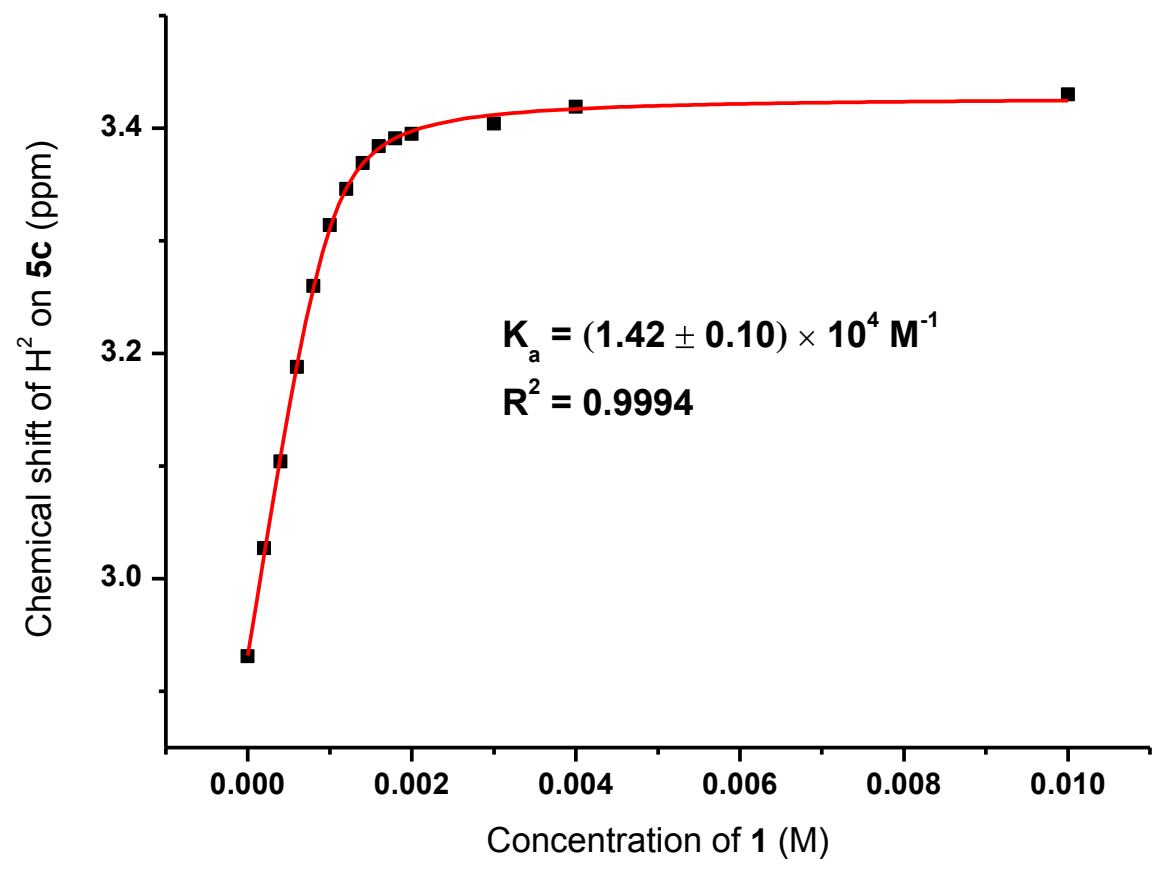

Figure 33. Determination of the binding constant of $\mathbf{1} \supset \mathbf{5 c}$ in $\mathrm{CDCl}_{3} / \mathrm{CD}_{3} \mathrm{CN}(1 / 1$, 
v/v) at 298 K. Fitting result based on $\mathrm{H}^{2}$ of $\mathbf{5 c}$.

\section{Molecular modelling of $2 \supset \mathrm{Tr}^{+}$}

The CT complex structure $\mathbf{2} \supset \mathbf{T r}^{+}$was optimized by the density functional theory (DFT) method at the RB3PW91/6-31G (d, p) level by employing the Gaussian09 program. $^{\text {S4 }}$
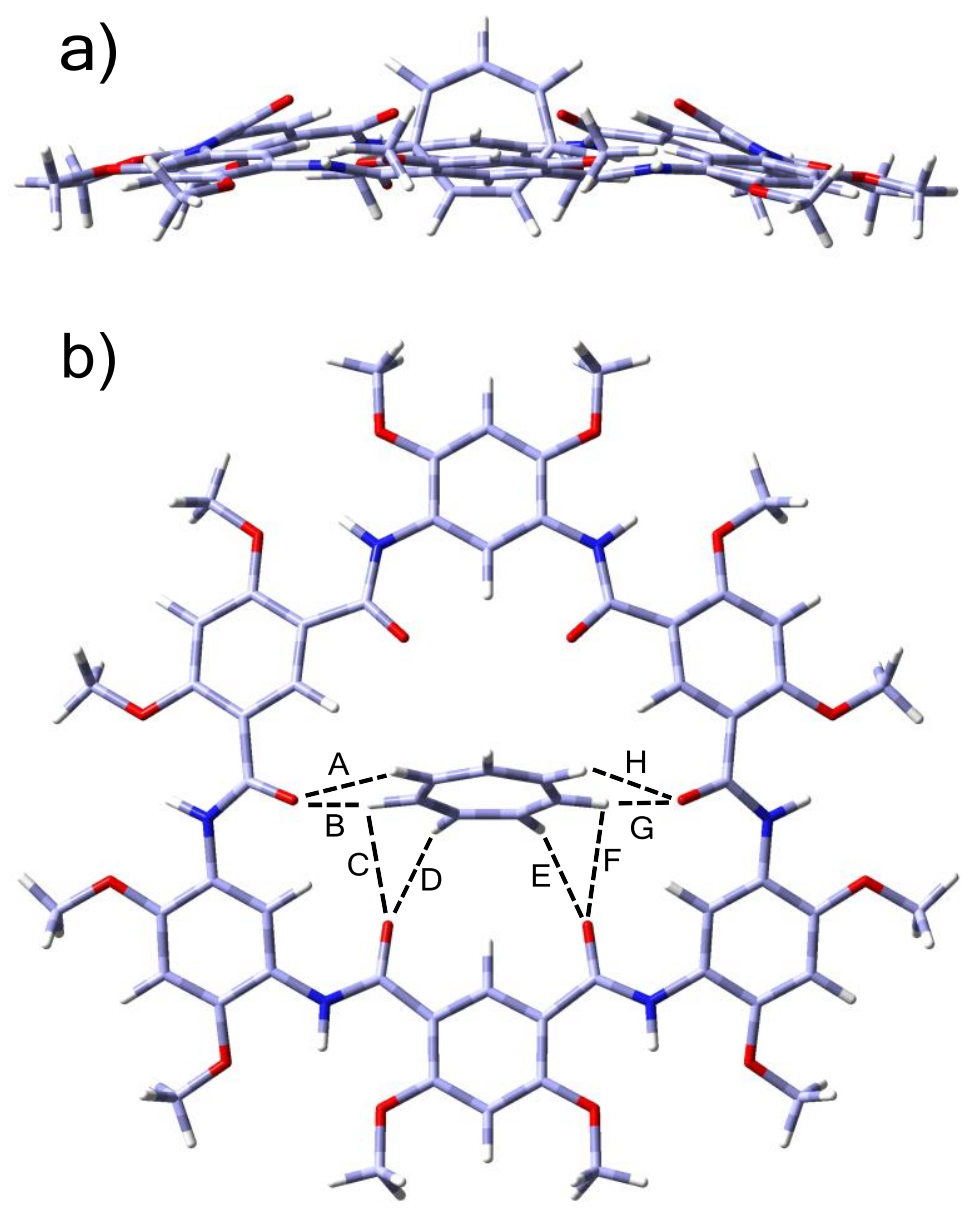

Figure 34. Side view a) and top view b) of optimized geometry of $\mathbf{2} \supset \mathbf{T r}^{+}$at the RB3PW91/6-31G (d, p) level. The plane of $\mathbf{T r}^{+}$is vertical to cyclo[6]aramide (gray = $\mathrm{C}$, white $=\mathrm{H}$, red $=\mathrm{O}$ and blue $=\mathrm{N}$ ). All side chains are replaced by methyl groups for simplicity. The dashed black lines indicate intermolecular $\mathrm{H}$-bonds $\mathrm{A}-\mathrm{H}$ and with $\mathrm{A}=2.531 \AA\left(118.2^{\circ}\right), \mathrm{B}=2.162 \AA\left(134.5^{\circ}\right), \mathrm{C}=2.848 \AA\left(90.6^{\circ}\right), \mathrm{D}=2.680 \AA\left(94.7^{\circ}\right)$, $\mathrm{E}=2.680 \AA\left(94.7^{\circ}\right), \mathrm{F}=2.848 \AA\left(90.6^{\circ}\right), \mathrm{G}=2.162 \AA\left(134.5^{\circ}\right), \mathrm{H}=2.531 \AA$ $\left(118.2^{\circ}\right)$.

Table S1. Atomic coordinates for the optimized structure of the complex $2 \supset \mathbf{T r}^{+}$. The plane of $\mathbf{T r}^{+}$is vertical to $\mathbf{2}$.

\begin{tabular}{cccccc}
\hline \multirow{2}{*}{ Center Number } & \multirow{2}{*}{ Atomic Number } & \multirow{2}{*}{ Atomic type } & \multicolumn{3}{c}{ Coordinates (Angstroms) } \\
& & & X & Y & Z \\
\hline 1 & 6 & 0 & -1.216677 & 6.107565 & 0.112615 \\
& & S25 & & &
\end{tabular}




\begin{tabular}{|c|c|c|c|c|c|}
\hline 2 & 6 & 0 & 0.000111 & 5.436172 & 0.268156 \\
\hline 3 & 6 & 0 & 1.216915 & 6.107547 & 0.112672 \\
\hline 4 & 6 & 0 & 1.20761 & 7.482401 & -0.187729 \\
\hline 5 & 6 & 0 & 0.000146 & 8.163258 & -0.33383 \\
\hline 6 & 6 & 0 & -1.207335 & 7.482418 & -0.187794 \\
\hline 7 & 1 & 0 & 0.000096 & 4.388241 & 0.520746 \\
\hline 8 & 1 & 0 & 0.000159 & 9.219364 & -0.566038 \\
\hline 9 & 8 & 0 & 2.436107 & 8.066508 & -0.328535 \\
\hline 10 & 8 & 0 & -2.435816 & 8.066546 & -0.32866 \\
\hline 11 & 6 & 0 & 2.490083 & 9.451155 & -0.60243 \\
\hline 12 & 1 & 0 & 2.014491 & 10.03743 & 0.193815 \\
\hline 13 & 1 & 0 & 3.54811 & 9.712352 & -0.651123 \\
\hline 14 & 1 & 0 & 2.017021 & 9.692361 & -1.563053 \\
\hline 15 & 6 & 0 & -2.48975 & 9.451169 & -0.602683 \\
\hline 16 & 1 & 0 & -3.547769 & 9.712378 & -0.651483 \\
\hline 17 & 1 & 0 & -2.014213 & 10.03751 & 0.193549 \\
\hline 18 & 1 & 0 & -2.016607 & 9.692281 & -1.563288 \\
\hline 19 & 7 & 0 & -2.479506 & 5.492806 & 0.188531 \\
\hline 20 & 6 & 0 & -2.753015 & 4.207087 & 0.548324 \\
\hline 21 & 1 & 0 & -3.263173 & 6.075823 & -0.079735 \\
\hline 22 & 8 & 0 & -1.895663 & 3.424703 & 0.95501 \\
\hline 23 & 6 & 0 & -4.172977 & 3.717875 & 0.384061 \\
\hline 24 & 6 & 0 & -5.313827 & 4.412518 & -0.067538 \\
\hline 25 & 6 & 0 & -4.340144 & 2.365694 & 0.658111 \\
\hline 26 & 6 & 0 & -6.528946 & 3.738282 & -0.246406 \\
\hline 27 & 6 & 0 & -5.51619 & 1.646148 & 0.472014 \\
\hline 28 & 1 & 0 & -3.470168 & 1.835426 & 1.017056 \\
\hline 29 & 6 & 0 & -6.63186 & 2.364899 & 0.000142 \\
\hline 30 & 1 & 0 & -7.396136 & 4.285011 & -0.589532 \\
\hline 31 & 8 & 0 & -5.179765 & 5.73914 & -0.316685 \\
\hline 32 & 6 & 0 & -6.293536 & 6.48578 & -0.778988 \\
\hline 33 & 1 & 0 & -6.653752 & 6.10882 & -1.743067 \\
\hline 34 & 1 & 0 & -7.109314 & 6.475747 & -0.047338 \\
\hline 35 & 1 & 0 & -5.933598 & 7.506729 & -0.902994 \\
\hline 36 & 8 & 0 & -7.781785 & 1.669427 & -0.190303 \\
\hline 37 & 6 & 0 & -8.938745 & 2.345846 & -0.656319 \\
\hline 38 & 1 & 0 & -9.716764 & 1.58636 & -0.730717 \\
\hline 39 & 1 & 0 & -9.256237 & 3.120109 & 0.051105 \\
\hline 40 & 1 & 0 & -8.769915 & 2.791224 & -1.643562 \\
\hline 41 & 6 & 0 & -5.39011 & 0.172689 & 0.730941 \\
\hline 42 & 8 & 0 & -4.398396 & -0.24791 & 1.334285 \\
\hline 43 & 7 & 0 & -6.324098 & -0.66255 & 0.197474 \\
\hline 44 & 1 & 0 & -7.175261 & -0.24718 & -0.161147 \\
\hline 45 & 6 & 0 & -6.205604 & -2.06145 & 0.076284 \\
\hline
\end{tabular}




\begin{tabular}{|c|c|c|c|c|c|}
\hline 46 & 6 & 0 & -7.370023 & -2.79799 & -0.20006 \\
\hline 47 & 6 & 0 & -4.982373 & -2.73083 & 0.132356 \\
\hline 48 & 6 & 0 & -7.296529 & -4.17945 & -0.39207 \\
\hline 49 & 6 & 0 & -4.888123 & -4.10301 & -0.091074 \\
\hline 50 & 1 & 0 & -4.086984 & -2.17944 & 0.353954 \\
\hline 51 & 6 & 0 & -6.06454 & -4.83362 & -0.336481 \\
\hline 52 & 1 & 0 & -8.197398 & -4.74362 & -0.591171 \\
\hline 53 & 7 & 0 & 2.479731 & 5.492764 & 0.188613 \\
\hline 54 & 6 & 0 & 2.753217 & 4.207052 & 0.548454 \\
\hline 55 & 1 & 0 & 3.263408 & 6.075748 & -0.079695 \\
\hline 56 & 8 & 0 & 1.895865 & 3.424716 & 0.955228 \\
\hline 57 & 6 & 0 & 4.173148 & 3.717782 & 0.3841 \\
\hline 58 & 6 & 0 & 5.314002 & 4.412386 & -0.067548 \\
\hline 59 & 6 & 0 & 4.340267 & 2.365585 & 0.65809 \\
\hline 60 & 6 & 0 & 6.529073 & 3.738095 & -0.246529 \\
\hline 61 & 6 & 0 & 5.516264 & 1.645984 & 0.471884 \\
\hline 62 & 1 & 0 & 3.470292 & 1.835344 & 1.017075 \\
\hline 63 & 6 & 0 & 6.631935 & 2.364696 & -0.000045 \\
\hline 64 & 1 & 0 & 7.396268 & 4.284796 & -0.589687 \\
\hline 65 & 8 & 0 & 5.179994 & 5.739027 & -0.316617 \\
\hline 66 & 6 & 0 & 6.293775 & 6.485635 & -0.778944 \\
\hline 67 & 1 & 0 & 7.109594 & 6.475519 & -0.047341 \\
\hline 68 & 1 & 0 & 6.653917 & 6.108711 & -1.743064 \\
\hline 69 & 1 & 0 & 5.933883 & 7.50661 & -0.902872 \\
\hline 70 & 8 & 0 & 7.781814 & 1.669177 & -0.1906 \\
\hline 71 & 6 & 0 & 8.938748 & 2.345543 & -0.656758 \\
\hline 72 & 1 & 0 & 9.256384 & 3.119766 & 0.050645 \\
\hline 73 & 1 & 0 & 9.716712 & 1.586013 & -0.731288 \\
\hline 74 & 1 & 0 & 8.769804 & 2.79096 & -1.643962 \\
\hline 75 & 6 & 0 & 5.390115 & 0.172523 & 0.730769 \\
\hline 76 & 8 & 0 & 4.398392 & -0.24803 & 1.334133 \\
\hline 77 & 7 & 0 & 6.324066 & -0.66275 & 0.197298 \\
\hline 78 & 1 & 0 & 7.175243 & -0.24742 & -0.161333 \\
\hline 79 & 6 & 0 & 6.205516 & -2.06166 & 0.076153 \\
\hline 80 & 6 & 0 & 4.98224 & -2.73097 & 0.132127 \\
\hline 81 & 6 & 0 & 7.369928 & -2.79828 & -0.200002 \\
\hline 82 & 6 & 0 & 4.887942 & -4.10316 & -0.091185 \\
\hline 83 & 1 & 0 & 4.086852 & -2.17952 & 0.353574 \\
\hline 84 & 6 & 0 & 7.296378 & -4.17975 & -0.391925 \\
\hline 85 & 6 & 0 & 6.064349 & -4.83386 & -0.336413 \\
\hline 86 & 1 & 0 & 8.197237 & -4.74399 & -0.590889 \\
\hline 87 & 8 & 0 & 8.522615 & -2.07178 & -0.276714 \\
\hline 88 & 8 & 0 & 5.90033 & -6.17839 & -0.502529 \\
\hline 89 & 6 & 0 & 9.726033 & -2.753 & -0.570264 \\
\hline
\end{tabular}




\begin{tabular}{|c|c|c|c|c|c|}
\hline 90 & 1 & 0 & 9.965221 & -3.49664 & 0.200087 \\
\hline 91 & 1 & 0 & 9.683072 & -3.24511 & -1.550208 \\
\hline 92 & 1 & 0 & 10.509362 & -1.99411 & -0.584978 \\
\hline 93 & 6 & 0 & 7.046049 & -6.96732 & -0.753682 \\
\hline 94 & 1 & 0 & 6.691885 & -7.99364 & -0.858695 \\
\hline 95 & 1 & 0 & 7.545838 & -6.66599 & -1.682803 \\
\hline 96 & 1 & 0 & 7.761501 & -6.9172 & 0.076909 \\
\hline 97 & 7 & 0 & 3.669322 & -4.8072 & -0.050556 \\
\hline 98 & 6 & 0 & 2.429966 & -4.27751 & -0.265154 \\
\hline 99 & 1 & 0 & 3.734297 & -5.81315 & 0.044928 \\
\hline 100 & 8 & 0 & 2.25301 & -3.08583 & -0.523583 \\
\hline 101 & 6 & 0 & 1.23637 & -5.18932 & -0.147409 \\
\hline 102 & 6 & 0 & 1.216696 & -6.5825 & 0.07436 \\
\hline 103 & 6 & 0 & -0.000103 & -4.55613 & -0.253578 \\
\hline 104 & 6 & 0 & -0.000122 & -7.26387 & 0.179099 \\
\hline 105 & 6 & 0 & -1.236585 & -5.18929 & -0.147342 \\
\hline 106 & 1 & 0 & -0.000094 & -3.48722 & -0.423557 \\
\hline 107 & 6 & 0 & -1.216931 & -6.58247 & 0.074429 \\
\hline 108 & 1 & 0 & -0.000129 & -8.33182 & 0.344076 \\
\hline 109 & 8 & 0 & 2.410142 & -7.22042 & 0.176018 \\
\hline 110 & 8 & 0 & -2.410381 & -7.22037 & 0.176158 \\
\hline 111 & 6 & 0 & 2.444937 & -8.6191 & 0.41225 \\
\hline 112 & 1 & 0 & 1.959497 & -8.87407 & 1.361054 \\
\hline 113 & 1 & 0 & 1.973485 & -9.17207 & -0.408044 \\
\hline 114 & 1 & 0 & 3.50076 & -8.88363 & 0.46537 \\
\hline 115 & 6 & 0 & -2.445183 & -8.61905 & 0.412389 \\
\hline 116 & 1 & 0 & -1.973783 & -9.17203 & -0.407933 \\
\hline 117 & 1 & 0 & -1.959695 & -8.87403 & 1.361165 \\
\hline 118 & 1 & 0 & -3.501007 & -8.88356 & 0.465567 \\
\hline 119 & 7 & 0 & -3.669531 & -4.8071 & -0.050422 \\
\hline 120 & 6 & 0 & -2.430161 & -4.27745 & -0.265034 \\
\hline 121 & 1 & 0 & -3.734539 & -5.81305 & 0.045058 \\
\hline 122 & 8 & 0 & -2.253178 & -3.08577 & -0.523455 \\
\hline 123 & 8 & 0 & -8.522659 & -2.07142 & -0.276859 \\
\hline 124 & 8 & 0 & -5.900576 & -6.17815 & -0.502687 \\
\hline 125 & 6 & 0 & -9.72606 & -2.75252 & -0.570739 \\
\hline 126 & 1 & 0 & -9.68292 & -3.24453 & -1.550725 \\
\hline 127 & 1 & 0 & -9.965465 & -3.49622 & 0.199481 \\
\hline 128 & 1 & 0 & -10.50933 & -1.99358 & -0.585537 \\
\hline 129 & 6 & 0 & -7.046306 & -6.96699 & -0.754077 \\
\hline 130 & 1 & 0 & -7.761865 & -6.91693 & 0.076424 \\
\hline 131 & 1 & 0 & -7.545953 & -6.66554 & -1.683231 \\
\hline 132 & 1 & 0 & -6.692185 & -7.99332 & -0.859154 \\
\hline 133 & 6 & 0 & -1.251927 & 0.218673 & 1.590757 \\
\hline
\end{tabular}




\begin{tabular}{cccccc}
134 & 6 & 0 & 0.000013 & 0.429353 & 2.163855 \\
135 & 6 & 0 & 1.251931 & 0.218701 & 1.590699 \\
136 & 6 & 0 & -1.556001 & -0.22605 & 0.305209 \\
137 & 6 & 0 & 1.555959 & -0.226 & 0.305132 \\
138 & 6 & 0 & -0.695412 & -0.56377 & -0.737389 \\
139 & 6 & 0 & 0.69533 & -0.56375 & -0.737425 \\
140 & 1 & 0 & -2.119007 & 0.408387 & 2.217624 \\
141 & 1 & 0 & 0.000034 & 0.792353 & 3.188533 \\
142 & 1 & 0 & 2.119034 & 0.408427 & 2.21753 \\
143 & 1 & 0 & -2.617621 & -0.33353 & 0.110814 \\
144 & 1 & 0 & 2.617574 & -0.33344 & 0.110689 \\
145 & 1 & 0 & -1.178323 & -0.904 & -1.648236 \\
146 & 1 & 0 & 1.178205 & -0.90396 & -1.648299 \\
\hline
\end{tabular}

The total electronic energy is calculated to be -4042.09287661 a. u..
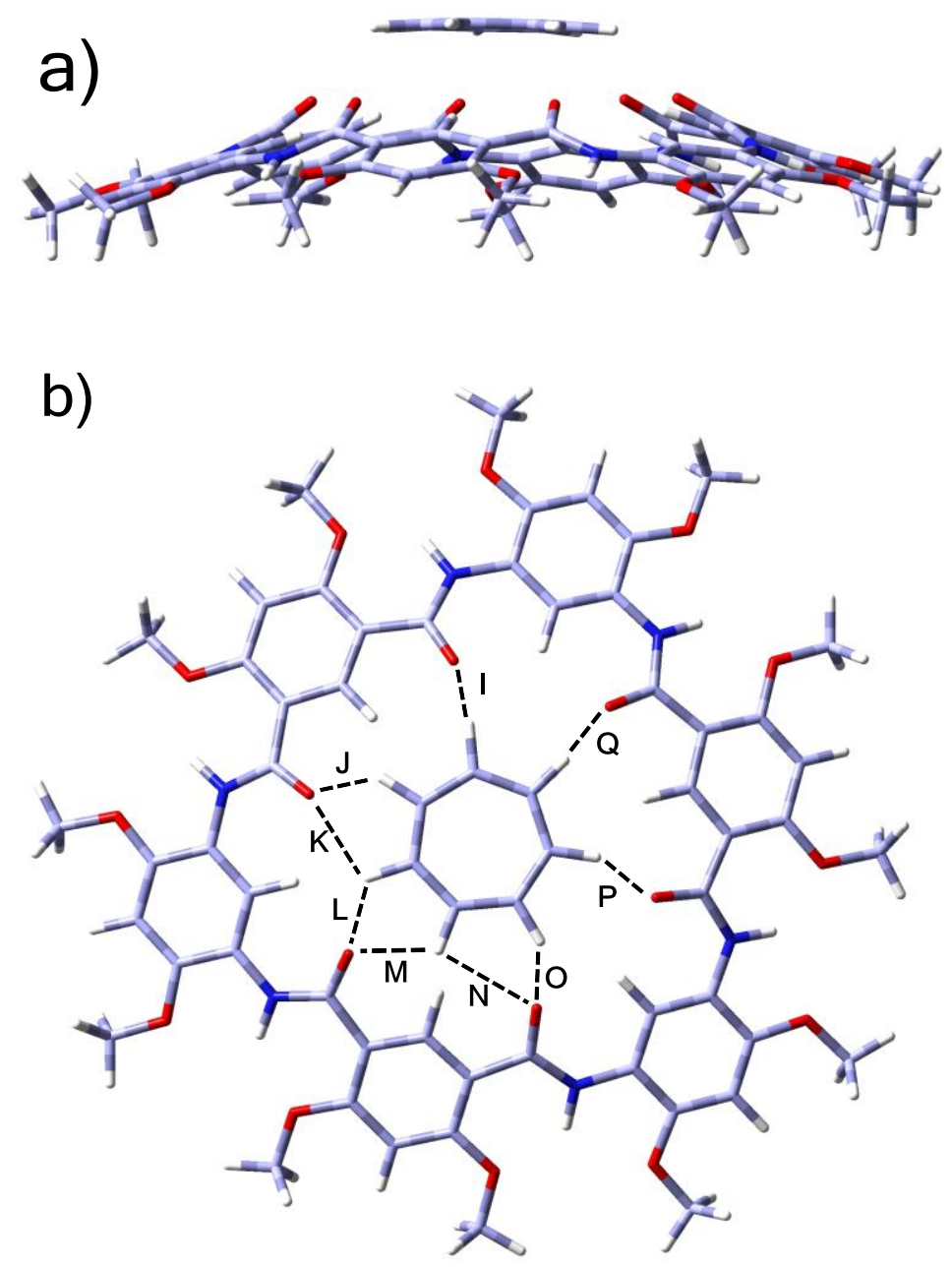

Figure 35. Side view a) and top view b) of optimized geometry of $\mathbf{2} \supset \mathbf{T r}^{+}$at the RB3PW91/6-31G (d, p) level. The plane of $\mathbf{T r}^{+}$is parallel to cyclo[6]aramide (gray = $\mathrm{C}$, white $=\mathrm{H}$, red $=\mathrm{O}$ and blue $=\mathrm{N}$ ). All side chains are replaced by methyl groups for simplicity. The dashed black lines indicate intermolecular H-bonds I-Q and with I $=2.220 \AA\left(137.8^{\circ}\right), \mathrm{J}=2.338 \AA\left(123.0^{\circ}\right), \mathrm{K}=2.859 \AA\left(105.5^{\circ}\right), \mathrm{L}=2.464 \AA\left(115.8^{\circ}\right)$, 
$\mathrm{M}=2.680 \AA\left(108.6^{\circ}\right), \mathrm{N}=3.173 \AA\left(98.7^{\circ}\right), \mathrm{O}=2.303 \AA\left(127.1^{\circ}\right), \mathrm{P}=2.243 \AA$ $\left(136.7^{\circ}\right), \mathrm{Q}=2.206 \AA\left(143.1^{\circ}\right)$.

Table S2. Atomic coordinates for the optimized structure of the complex $\mathbf{2} \supset \mathbf{T r}^{+}$. The plane of $\mathbf{T r}^{+}$is parallel to $\mathbf{2}$.

\begin{tabular}{|c|c|c|c|c|c|}
\hline \multirow{2}{*}{ Center Number } & \multirow{2}{*}{ Atomic Number } & \multirow{2}{*}{ Atomic type } & \multicolumn{3}{|c|}{ Coordinates (Angstroms) } \\
\hline & & & $\mathrm{X}$ & $\mathrm{Y}$ & $\mathrm{Z}$ \\
\hline 1 & 6 & 0 & 0.626182 & 6.324102 & -0.14577 \\
\hline 2 & 6 & 0 & 1.604315 & 5.354492 & 0.083101 \\
\hline 3 & 6 & 0 & 2.957269 & 5.631859 & -0.12076 \\
\hline 4 & 6 & 0 & 3.335893 & 6.916264 & -0.55216 \\
\hline 5 & 6 & 0 & 2.367922 & 7.895358 & -0.77585 \\
\hline 6 & 6 & 0 & 1.018429 & 7.604248 & -0.57756 \\
\hline 7 & 1 & 0 & 1.310401 & 4.37678 & 0.42679 \\
\hline 8 & 1 & 0 & 2.663986 & 8.880792 & -1.10871 \\
\hline 9 & 8 & 0 & 4.675707 & 7.105562 & -0.74351 \\
\hline 10 & 8 & 0 & 0.002752 & 8.492641 & -0.79443 \\
\hline 11 & 6 & 0 & 5.11261 & 8.36778 & -1.20553 \\
\hline 12 & 1 & 0 & 4.861104 & 9.165201 & -0.49501 \\
\hline 13 & 1 & 0 & 6.197874 & 8.301712 & -1.29422 \\
\hline 14 & 1 & 0 & 4.685791 & 8.607956 & -2.18774 \\
\hline 15 & 6 & 0 & 0.333277 & 9.783898 & -1.26412 \\
\hline 16 & 1 & 0 & -0.61059 & 10.31956 & -1.37478 \\
\hline 17 & 1 & 0 & 0.96667 & 10.32266 & -0.54824 \\
\hline 18 & 1 & 0 & 0.838697 & 9.742205 & -2.23747 \\
\hline 19 & 7 & 0 & -0.75514 & 6.080412 & -0.04658 \\
\hline 20 & 6 & 0 & -1.35694 & 4.975553 & 0.474935 \\
\hline 21 & 1 & 0 & -1.35672 & 6.800798 & -0.4282 \\
\hline 22 & 8 & 0 & -0.73569 & 4.089643 & 1.063685 \\
\hline 23 & 6 & 0 & -2.83454 & 4.80712 & 0.245876 \\
\hline 24 & 6 & 0 & -3.76562 & 5.762842 & -0.20844 \\
\hline 25 & 6 & 0 & -3.31505 & 3.521208 & 0.477545 \\
\hline 26 & 6 & 0 & -5.10315 & 5.405388 & -0.40899 \\
\hline 27 & 6 & 0 & -4.63088 & 3.114928 & 0.274915 \\
\hline 28 & 1 & 0 & -2.60404 & 2.777607 & 0.81306 \\
\hline 29 & 6 & 0 & -5.53531 & 4.094531 & -0.18337 \\
\hline 30 & 1 & 0 & -5.81 & 6.149671 & -0.74772 \\
\hline 31 & 8 & 0 & -3.30949 & 7.021521 & -0.43145 \\
\hline 32 & 6 & 0 & -4.20454 & 8.023738 & -0.88717 \\
\hline 33 & 1 & 0 & -4.63248 & 7.763473 & -1.86232 \\
\hline 34 & 1 & 0 & -5.008 & 8.196545 & -0.16225 \\
\hline 35 & 1 & 0 & -3.60749 & 8.930182 & -0.98488 \\
\hline 36 & 8 & 0 & -6.82161 & 3.710202 & -0.3843 \\
\hline 37 & 6 & 0 & -7.77486 & 4.656565 & -0.84094 \\
\hline
\end{tabular}




\begin{tabular}{|c|c|c|c|c|c|}
\hline 38 & 1 & 0 & -8.71539 & 4.112163 & -0.92239 \\
\hline 39 & 1 & 0 & -7.89306 & 5.476979 & -0.1241 \\
\hline 40 & 1 & 0 & -7.50046 & 5.057585 & -1.82362 \\
\hline 41 & 6 & 0 & -4.88934 & 1.65501 & 0.531987 \\
\hline 42 & 8 & 0 & -4.05738 & 0.992834 & 1.15464 \\
\hline 43 & 7 & 0 & -6.01464 & 1.111388 & -0.00998 \\
\hline 44 & 1 & 0 & -6.69543 & 1.751649 & -0.40104 \\
\hline 45 & 6 & 0 & -6.33825 & -0.25357 & -0.10296 \\
\hline 46 & 6 & 0 & -7.64883 & -0.57191 & -0.50297 \\
\hline 47 & 6 & 0 & -5.41662 & -1.28426 & 0.091131 \\
\hline 48 & 6 & 0 & -8.01838 & -1.90167 & -0.70427 \\
\hline 49 & 6 & 0 & -5.77362 & -2.61834 & -0.11359 \\
\hline 50 & 1 & 0 & -4.41361 & -1.04612 & 0.404669 \\
\hline 51 & 6 & 0 & -7.08768 & -2.92263 & -0.51331 \\
\hline 52 & 1 & 0 & -9.02652 & -2.14109 & -1.01393 \\
\hline 53 & 7 & 0 & 3.978119 & 4.672771 & 0.000579 \\
\hline 54 & 6 & 0 & 3.872681 & 3.422443 & 0.531391 \\
\hline 55 & 1 & 0 & 4.880888 & 4.943138 & -0.37153 \\
\hline 56 & 8 & 0 & 2.863744 & 3.025897 & 1.116442 \\
\hline 57 & 6 & 0 & 5.018258 & 2.472269 & 0.309258 \\
\hline 58 & 6 & 0 & 6.324744 & 2.766022 & -0.1312 \\
\hline 59 & 6 & 0 & 4.71363 & 1.130627 & 0.522229 \\
\hline 60 & 6 & 0 & 7.248761 & 1.736676 & -0.33775 \\
\hline 61 & 6 & 0 & 5.59217 & 0.071639 & 0.30998 \\
\hline 62 & 1 & 0 & 3.709421 & 0.892053 & 0.847605 \\
\hline 63 & 6 & 0 & 6.889284 & 0.400528 & -0.13396 \\
\hline 64 & 1 & 0 & 8.25114 & 1.976299 & -0.66363 \\
\hline 65 & 8 & 0 & 6.635944 & 4.071479 & -0.33533 \\
\hline 66 & 6 & 0 & 7.935236 & 4.4231 & -0.78365 \\
\hline 67 & 1 & 0 & 8.699479 & 4.121898 & -0.05828 \\
\hline 68 & 1 & 0 & 8.154123 & 3.978333 & -1.76147 \\
\hline 69 & 1 & 0 & 7.93249 & 5.509222 & -0.8727 \\
\hline 70 & 8 & 0 & 7.755806 & -0.62319 & -0.34401 \\
\hline 71 & 6 & 0 & 9.068273 & -0.3495 & -0.80814 \\
\hline 72 & 1 & 0 & 9.624622 & 0.260527 & -0.08753 \\
\hline 73 & 1 & 0 & 9.554098 & -1.31987 & -0.90814 \\
\hline 74 & 1 & 0 & 9.050026 & 0.150565 & -1.78354 \\
\hline 75 & 6 & 0 & 5.002133 & -1.29414 & 0.53655 \\
\hline 76 & 8 & 0 & 3.928103 & -1.39851 & 1.131288 \\
\hline 77 & 7 & 0 & 5.658293 & -2.36064 & -0.00031 \\
\hline 78 & 1 & 0 & 6.585546 & -2.19333 & -0.37275 \\
\hline 79 & 6 & 0 & 5.183667 & -3.67921 & -0.11499 \\
\hline 80 & 6 & 0 & 3.847269 & -4.0399 & 0.06632 \\
\hline 81 & 6 & 0 & 6.109366 & -4.65894 & -0.5172 \\
\hline
\end{tabular}




\begin{tabular}{|c|c|c|c|c|c|}
\hline 82 & 6 & 0 & 3.417164 & -5.35003 & -0.15295 \\
\hline 83 & 1 & 0 & 3.137022 & -3.29558 & 0.385613 \\
\hline 84 & 6 & 0 & 5.692529 & -5.97258 & -0.73265 \\
\hline 85 & 6 & 0 & 4.353584 & -6.3203 & -0.554 \\
\hline 86 & 1 & 0 & 6.407623 & -6.72171 & -1.04409 \\
\hline 87 & 8 & 0 & 7.393243 & -4.22432 & -0.68967 \\
\hline 88 & 8 & 0 & 3.85272 & -7.57465 & -0.76142 \\
\hline 89 & 6 & 0 & 8.364611 & -5.16022 & -1.11114 \\
\hline 90 & 1 & 0 & 8.481293 & -5.97362 & -0.38403 \\
\hline 91 & 1 & 0 & 8.118878 & -5.58311 & -2.09371 \\
\hline 92 & 1 & 0 & 9.305199 & -4.61247 & -1.18359 \\
\hline 93 & 6 & 0 & 4.742178 & -8.58866 & -1.18346 \\
\hline 94 & 1 & 0 & 4.144887 & -9.49588 & -1.28473 \\
\hline 95 & 1 & 0 & 5.197694 & -8.3489 & -2.1528 \\
\hline 96 & 1 & 0 & 5.533053 & -8.76336 & -0.44326 \\
\hline 97 & 7 & 0 & 2.073027 & -5.75541 & -0.07347 \\
\hline 98 & 6 & 0 & 1.031058 & -5.04034 & 0.434402 \\
\hline 99 & 1 & 0 & 1.86586 & -6.67055 & -0.4554 \\
\hline 100 & 8 & 0 & 1.176739 & -3.97023 & 1.026554 \\
\hline 101 & 6 & 0 & -0.36101 & -5.55636 & 0.191164 \\
\hline 102 & 6 & 0 & -0.76064 & -6.82795 & -0.26608 \\
\hline 103 & 6 & 0 & -1.37008 & -4.62591 & 0.421501 \\
\hline 104 & 6 & 0 & -2.11588 & -7.11197 & -0.46607 \\
\hline 105 & 6 & 0 & -2.72777 & -4.85745 & 0.224482 \\
\hline 106 & 1 & 0 & -1.07157 & -3.64023 & 0.752953 \\
\hline 107 & 6 & 0 & -3.09383 & -6.13878 & -0.23495 \\
\hline 108 & 1 & 0 & -2.41063 & -8.09478 & -0.80588 \\
\hline 109 & 8 & 0 & 0.214891 & -7.74385 & -0.49339 \\
\hline 110 & 8 & 0 & -4.41583 & -6.37593 & -0.43163 \\
\hline 111 & 6 & 0 & -0.13144 & -9.03919 & -0.95687 \\
\hline 112 & 1 & 0 & -0.76777 & -9.56178 & -0.23377 \\
\hline 113 & 1 & 0 & -0.63369 & -8.99345 & -1.93018 \\
\hline 114 & 1 & 0 & 0.810621 & -9.57703 & -1.06131 \\
\hline 115 & 6 & 0 & -4.8399 & -7.64942 & -0.89127 \\
\hline 116 & 1 & 0 & -4.418 & -7.87856 & -1.87676 \\
\hline 117 & 1 & 0 & -4.57041 & -8.4373 & -0.17886 \\
\hline 118 & 1 & 0 & -5.92535 & -7.58949 & -0.96742 \\
\hline 119 & 7 & 0 & -4.86905 & -3.69131 & -0.03201 \\
\hline 120 & 6 & 0 & -3.61386 & -3.67237 & 0.497055 \\
\hline 121 & 1 & 0 & -5.1909 & -4.56703 & -0.42674 \\
\hline 122 & 8 & 0 & -3.16071 & -2.71214 & 1.121883 \\
\hline 123 & 8 & 0 & -8.4844 & 0.492738 & -0.69013 \\
\hline 124 & 8 & 0 & -7.35302 & -4.24843 & -0.70992 \\
\hline 125 & 6 & 0 & -9.80466 & 0.238072 & -1.12554 \\
\hline
\end{tabular}




\begin{tabular}{|c|c|c|c|c|c|}
\hline 126 & 1 & 0 & -9.81821 & -0.26195 & -2.10246 \\
\hline 127 & 1 & 0 & -10.3579 & -0.36939 & -0.39838 \\
\hline 128 & 1 & 0 & -10.2894 & 1.211129 & -1.21558 \\
\hline 129 & 6 & 0 & -8.64574 & -4.61324 & -1.14949 \\
\hline 130 & 1 & 0 & -9.41417 & -4.32496 & -0.42132 \\
\hline 131 & 1 & 0 & -8.88176 & -4.16647 & -2.12376 \\
\hline 132 & 1 & 0 & -8.63973 & -5.69972 & -1.24666 \\
\hline 133 & 6 & 0 & -1.65388 & -0.59361 & 2.802281 \\
\hline 134 & 6 & 0 & -0.6866 & -1.59834 & 2.81154 \\
\hline 135 & 6 & 0 & 0.702594 & -1.47414 & 2.772665 \\
\hline 136 & 6 & 0 & -1.4662 & 0.788941 & 2.783218 \\
\hline 137 & 6 & 0 & 1.469126 & -0.30811 & 2.721086 \\
\hline 138 & 6 & 0 & -0.27097 & 1.507637 & 2.738016 \\
\hline 139 & 6 & 0 & 1.037003 & 1.018798 & 2.704065 \\
\hline 140 & 1 & 0 & -2.68454 & -0.93378 & 2.759668 \\
\hline 141 & 1 & 0 & -1.07473 & -2.61245 & 2.802095 \\
\hline 142 & 1 & 0 & 1.255505 & -2.40726 & 2.716423 \\
\hline 143 & 1 & 0 & -2.38221 & 1.371105 & 2.74055 \\
\hline 144 & 1 & 0 & 2.540818 & -0.46709 & 2.627375 \\
\hline 145 & 1 & 0 & -0.37275 & 2.586894 & 2.657267 \\
\hline 146 & 1 & 0 & 1.815368 & 1.771283 & 2.597201 \\
\hline
\end{tabular}

The total electronic energy is calculated to be -4042.10425863 a.u..

Table S3. The result of calculated single point energy of $\mathbf{T r}^{+}$in the CT complex 2 د $\mathrm{Tr}^{+}$in parallel conformation at the RB3PW91/6-31G (d, p) level. Molecular unit 2 represents $\operatorname{Tr}^{+}\left(\mathrm{C}_{7} \mathrm{H}_{7}^{+}\right)$.

\begin{tabular}{|c|c|}
\hline Total Lewis & $44.31234 \quad(92.1979 \%)$ \\
\hline Valence non-Lewis & $3.66595(7.6275 \%)$ \\
\hline Rydberg non-Lewis & $0.08389(0.1745 \%)$ \\
\hline Total unit 2 & $48.06219 \quad(100.0000 \%)$ \\
\hline Charge unit 2 & 0.93781 \\
\hline
\end{tabular}

\section{References}

(S1).Hu, J. C.; Chen, L.; Ren, Y.; Deng, P. C.; Li, X. W.; Wang, Y. J.; Jia, Y. M.; Luo, J.; Yang, X. S.; Feng, W.; Yuan, L. H. Org. Lett. 2013, 15, 4670. 
(S2) Zhang, C. J.; Li, S. J.; Zhang, J. Q.; Zhu, K. L.; Li, N.; Huang, F. H. Org. Lett. 2007, 9, 5553.

(S3) Hu, J. C.; Chen, L.; Shen, J.; Luo, J.; Deng, P. C.; Ren, Y.; Zeng, H. Q.; Feng W.; Yuan, L. H. Chem. Commun. 2014, 50, 8024.

(S4) Gaussian 09, Revision B.01, Frisch, M.J.; Trucks, G.W.; Schlegel, H.B.; Scuseria, G.E.; Robb, M.A.; Cheeseman, J.R.; Scalmani, G.; Barone, V.; Mennucci, B.; Petersson, G.A.; Nakatsuji, H.; Caricato, M.; Li, X.; Hratchian, H. P.; Izmaylov, A. F.; Bloino, J.; Zheng, G.; Sonnenberg,J.L.; Hada, M.; Ehara, M.; Toyota, K.; Fukuda, R.; Hasegawa, J.; Ishida, M.; Nakajima, T.; Honda, Y.; Kitao, O.; Nakai, H.; Vreven, T.; Montgomery, J.A., Jr.; Peralta, J.E.; Ogliaro, F.; Bearpark, M.; Heyd, J. J.; Brothers, E.; Kudin, K. N.; Staroverov, V. N.; Keith, T.; Kobayashi, R.; Normand, J.; Raghavachari, K.; Rendell, A.; Burant, J. C.; Iyengar, S.S.; Tomasi, J.; Cossi, M.; Rega, N.; Millam, J.M.; Klene, M.; Knox, J.E.; Cross, J.B.; Bakken, V.; Adamo, C.; Jaramillo, J.; Gomperts, R.; Stratmann, R.E.; Yazyev, O.; Austin, A.J.; Cammi, R.; Pomelli, C.; Ochterski, J.W.; Martin, R.L.; Morokuma, K.; Zakrzewski, V.G.; Voth, G.A.; Salvador, P.; Dannenberg, J.J.; Dapprich, S.; Daniels, A.D.; Farkas, O.J.; Foresman, B.; Ortiz, J.V.; Cioslowski, J.; Fox, D. J. Gaussian, Inc., Wallingford CT, 2010. 